\title{
Automated Vision-based Loosened Bolt Detection using the Cascade Detector
}

\author{
By \\ Lovedeep Ramana
}

A thesis is submitted to the Faculty of Graduate Studies of University of Manitoba

In partial fulfillment of the requirements of the degree of

\section{Master of Science}

Department of Civil Engineering

Faculty of Engineering

University of Manitoba

Winnipeg

Copyright $\mathbb{C} 2017$ by Lovedeep Ramana 


\begin{abstract}
Numerous damage detection methods that use data obtained from contact sensors, physically attached to structures and human inspection methods have been developed. However, damage sensitive features used for these methods such as modal properties of steel and reinforced concrete structures are sensitive to environmental conditions such as temperature and humidity. Besides, human inspection is cost, labor extensive, and is controlled by the technical understanding of an individual. The uncertainties of the contact sensor methods are difficult to address with a regression model or any other temperature compensation method, and these are primary causes of false alarms. In order to address some of these challenges of the traditional sensing system, a vision-based remote sensing system can be one of the alternatives as it gives the explicit intuitions of structural conditions. In addition, bolted connections are common engineering practices, and very few vision-based techniques are developed for loosened bolt detection. Thus, this thesis proposes an automated vision-based method for detecting loosened structural bolts using the Viola-Jones algorithm and support vector machines. The test images of bolt connections are taken with a digital single lens reflex camera. The Viola-Jones algorithm is trained on two datasets of images with and without bolts. The trained algorithm localizes and crops all the bolts on test images. The SVM is trained on another dataset of loose and tight bolts to generate decision boundary for classification of the loosened and tight bolts. The cropped bolt images are binarized to calculate bolt features as head dimensions and exposed shank length. The extracted features are fed into a trained support vector machine to classify the loosened and tight bolts. We test our method on images taken by digital single lens reflex and smartphone cameras.
\end{abstract}


A special feeling of gratitude to my loving parents. Their words of encouragement, support, and prayers helped me to come this far. 


\section{Acknowledgements}

First and foremost, I would like to express my sincere appreciation and thankfulness to my advisor Dr. Young-Jin Cha, Assistant Professor, Structural Engineering in the Department of Civil Engineering at the University of Manitoba. His continuous guidance and financial support helped me throughout this work. The door to Dr. Cha's office was always open whenever I needed his assistance. He consistently steered me in the right direction whenever he thought I needed it.

Special thanks to my colleague, especially Mr. Wooram Choi, Ph.D student whose suggestions for my work were phenomenal. Special thanks to Mr. Tyler Epp and Mr. Darshan L. Gopal for knowledge bytes.

The help of W. R. McQuade structures laboratory technical staff, Dr. Chad Klowak, Peng, Brenden Pachal and Grant Whiteside is greatly appreciated.

Thanks to my friends, Tarun Bedi, Vishal Chaudhary, Ishan Dhiman, Harjot Singh, Puneet Anand and my girlfriend Nancy Sood for their unfailing support, and for being there for me, during both good and bad times.

Lastly, and most importantly, I would like to thank my father and my mother. Words cannot describe the sacrifices my parents have made for me, to assure a bright future; without them, I would not have been here. 


\section{Table of Contents}

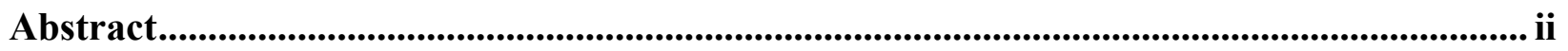

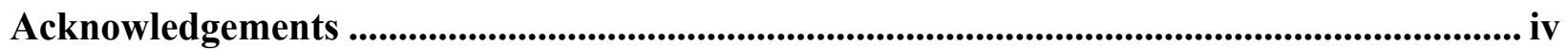

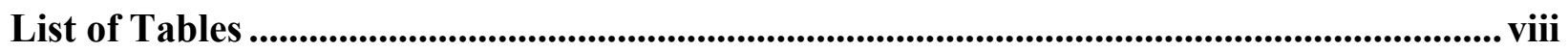

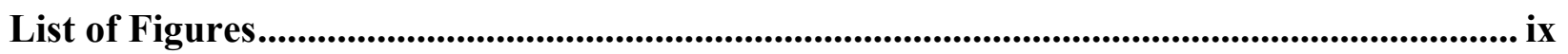

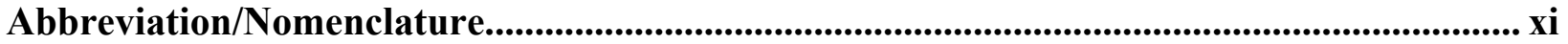

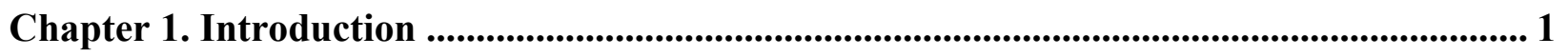

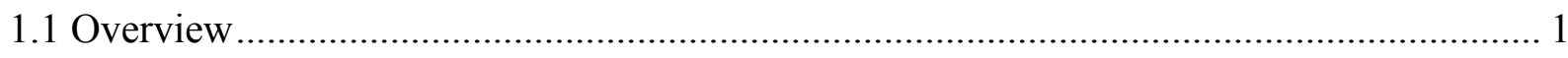

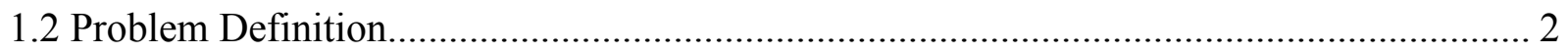

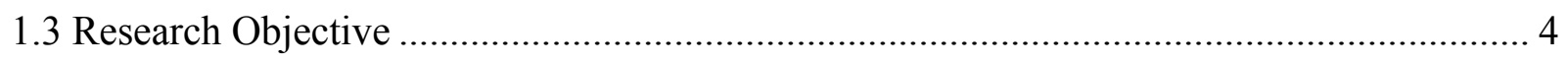

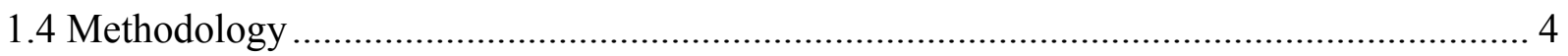

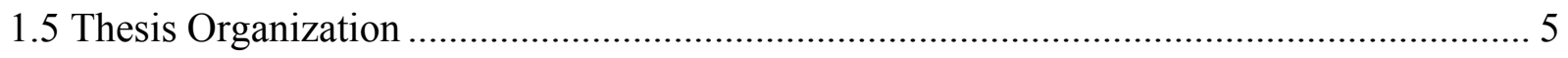

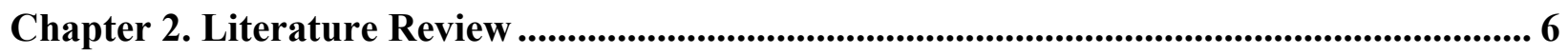

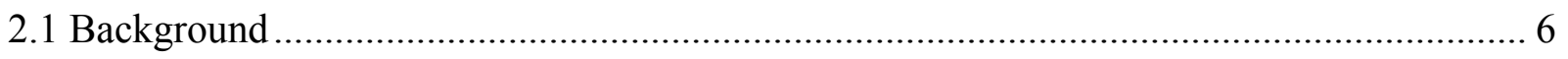

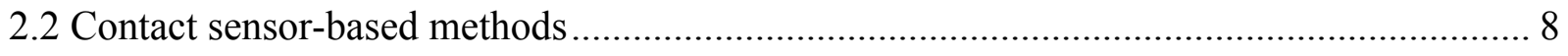

2.2.1 Contact sensor damage detection in concrete structures.................................... 9

2.2.2 Contact sensor damage detection in steel structures........................................ 11

2.3 Vision-based damage detection methods............................................................. 13

2.3.1 Crack detection by vision-based methods.................................................... 14

2.3.2 Corrosion detection by vision-based methods ................................................ 16 
2.3.3 Displacement by vision-based methods.................................................................. 17

2.3.4 Loosened bolt detection by vision-based methods .................................................. 18

Chapter 3 - Methodology of vision based damage detection for bolts....................................... 20

3.1 Overview of the vision based loosened bot detection method............................................ 20

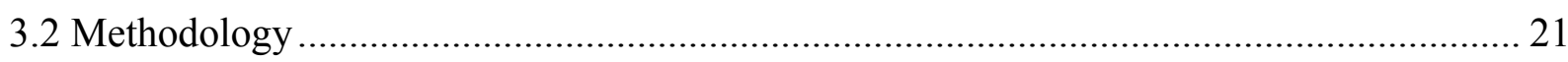

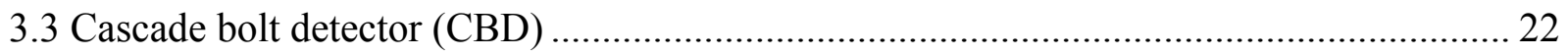

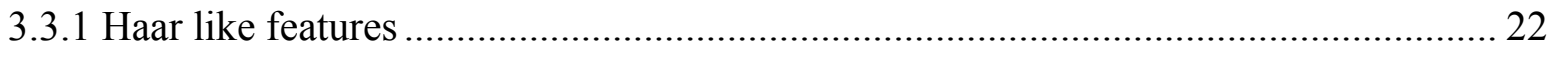

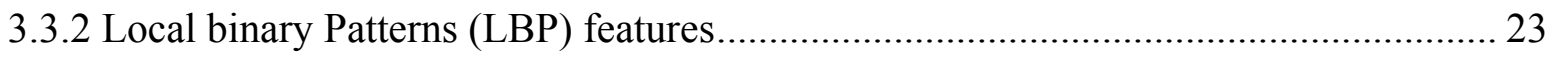

3.3.3 Histogram of oriented gradients (HOG) features...................................................... 24

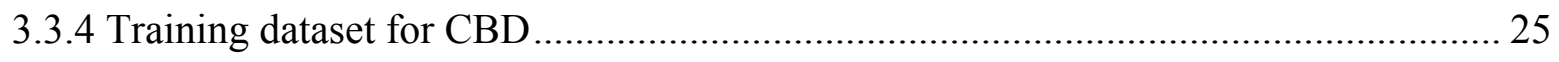

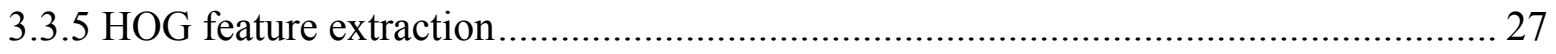

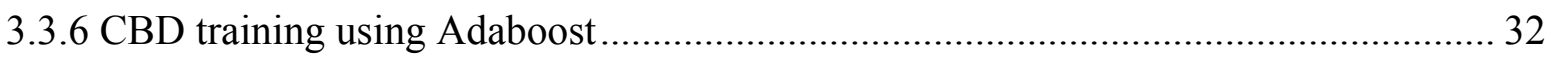

3.4 Support vector machine (SVM) classifier......................................................................... 38

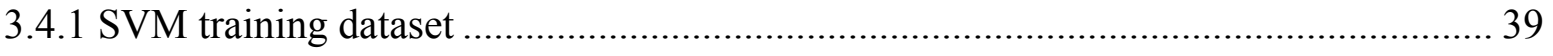

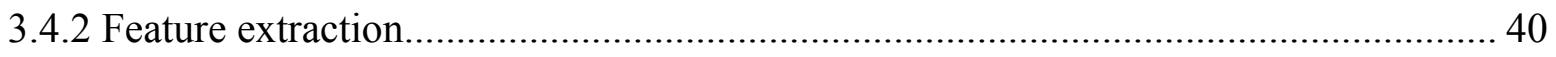

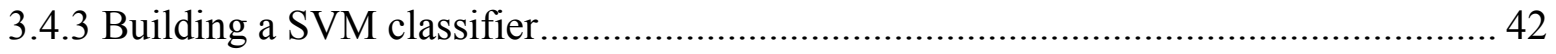

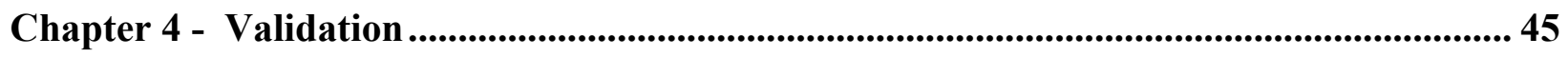

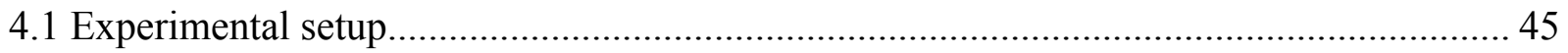

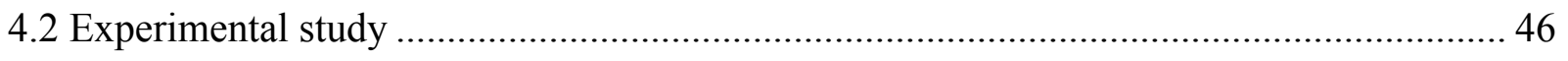

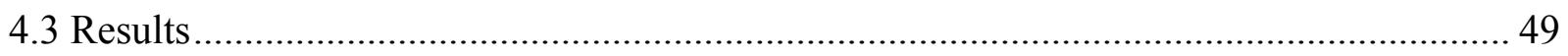

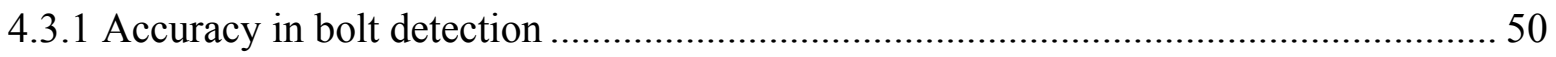

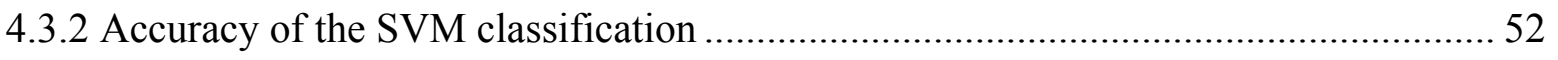

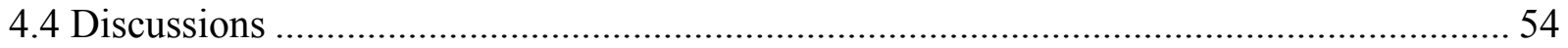


5.1 Summary

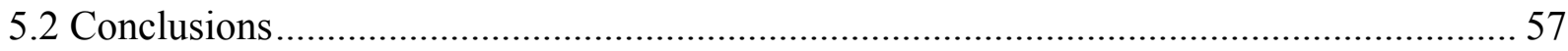

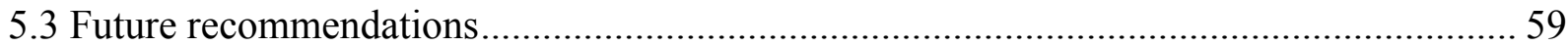

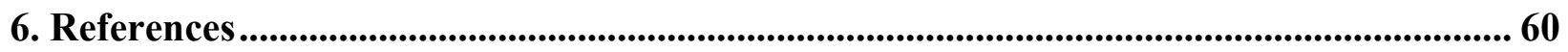

Appendix A 


\section{List of Tables}

Table 4.1. Images were taken at combination of vertical and horizontal angle........................ 46

Table 4.2. The number of bolts according to the range of vertical angles and loosened length... 49

Table 4.3. Accuracy of the CBD for loosened and tight dataset............................................ 51

Table 4.4. Accuracy of the SVM classification for the loosened and tight dataset.................... 53

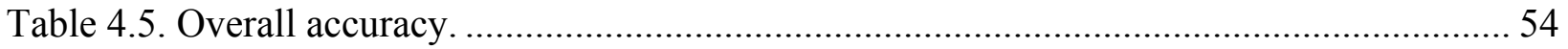




\section{List of Figures}

Figure 2.1. Types of defects in civil structures ............................................................... 7

Figure 2.2. A schematic diagram of sensor based damage detection method. ........................... 8

Figure 2.3. A basic process of image processing algorithm. ............................................ 14

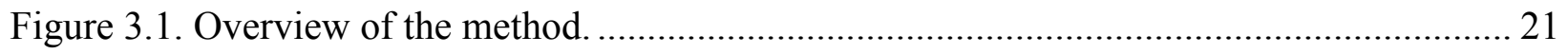

Figure 3.2. Haar features (a) two rectangle feature (b) three rectangle feature (c) four rectangle

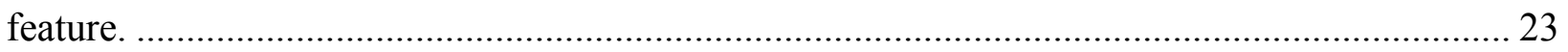

Figure 3.3. Training dataset for CBD, images with bolts are positive dataset; images without bolts

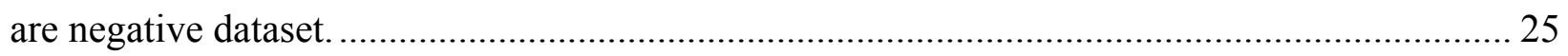

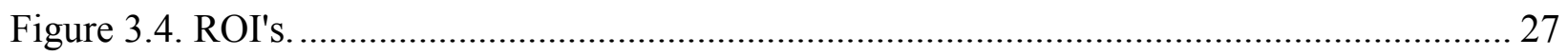

Figure 3.5. This section is focused on HOG feature extraction in CBD training...................... 27

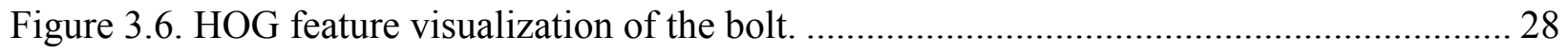

Figure 3.7. (a) A simplified view of image divided into smaller regions blocks and cells, (b) 50\% block overlapping in horizontal direction, (c) 50\% block overlapping in vertical direction........ 29 Figure 3.8. Explains the gradient and orientation calculations and interpolation for histogram

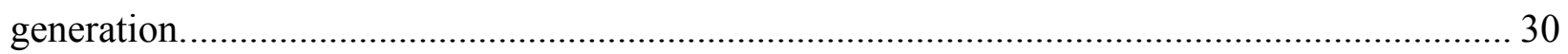

Figure 3.9. Visualization of the histogram of a cell..................................................... 31

Figure 3.10. This section refers to building classifier using Adaboost stage in training............. 32

Figure 3.11 - Simplified view of scanning window over the image for detecting bolts............ 33

Figure 3.12 - Schematic diagram explaining working of Adaboost ..................................... 34

Figure 3.13. Explains how sub windows proceed through different stages based on the bolt

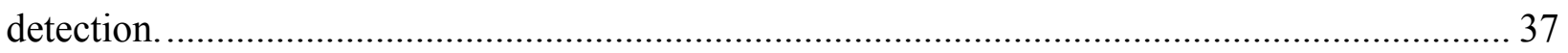


Figure 3.14. Illustration of SVM classifier with hyperplane differentiating data classes............ 38

Figure 3.15. Training dataset for SVM training. ............................................................ 40

Figure 3.16. (a) Represent bolt features used for classification (b) represent the ellipse detection over the bolt head (c) binary detector for calculating the vertical height of the bolt (h) and (d) shows the calculated bolt features from the ellipse parameters and binary detector.

Figure 3.17. Shows the contrast enhancement and masking of the binary image for accurate feature calculation. 42

Figure 3.18. Bolt feature comparison between loose bolts at farther distance and tight bolts at lesser distance. 43

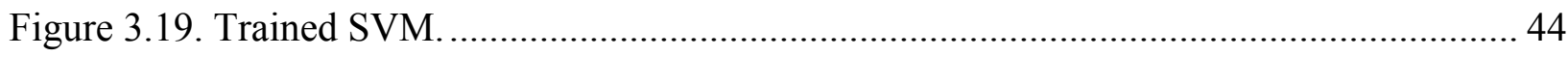

Figure 4.1. (a) Experimental setup (b) bolt details on the specimen. .................................... 45

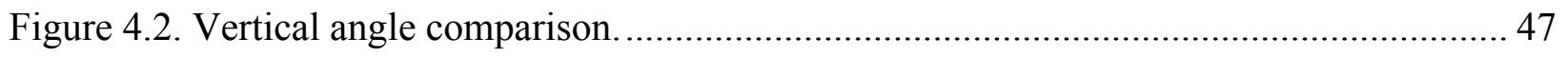

Figure 4.3. Effect on horizontal angle on bolt layout geometry......................................... 48

Figure 4.4. This section is focused on CBD accuracy .................................................. 50

Figure 4.5. Partial detections, shadows effect and false detections by CBD.......................... 51

Figure 4.6. This section is focused on SVM classification accuracy of images provided by CBD.

Figure 4.7. (a) False classification by SVM due to sharp vertical angle. (b) No bolt detection by CBD due to overlapping at low vertical angle 56 


\section{Abbreviation/Nomenclature}

American Society of Civil Engineers (ASCE)

structural health monitoring (SHM)

support vector machines (SVM's)

cascade bolt detector (CBD)

Lead Zirconate Titanate/piezoelectric (PZT)

electro-mechanical impedance (EMI)

convolution neural networks $(\mathrm{CNN})$

Fast Fourier Transform (FFT)

Histogram of oriented gradients (HOG)

or Local binary patterns (LBP)

digital single lens reflex DSLR

region of interest (ROI) 


\section{Chapter 1. Introduction}

\subsection{Overview}

Infrastructure around the world is growing. With an increase in population, the use of existing structures is becoming more stressful. Civil structures are designed for safe performance under varying environmental conditions and long-term public use. Throughout their life span, structures go through uncountable loading cycles and vibrations. As a result, the material and structural members such as beams, slabs, columns, cables, bolts, and joints are subject to deterioration with time, catastrophic events, or manmade disasters. Earthquakes, public use, and cyclic climatic changes etc., deteriorate the safety domain of the structures defined by the designers [1]. Due to the above-mentioned reasons, new structures might show signs of fatigue, which have to be recognized and examined for safe public use. In addition, existing structures are aging and passing their service lives. As per Canadian infrastructure report card of 2016, about one third of infrastructure owned by municipal corporations needs repairs. In Canada about $60 \%$ of infrastructure is under municipal corporations [2]. A 2017 American Society of Civil Engineers (ASCE) report rated United States infrastructure at grade D+: a structure at risk [3]. ASCE traces the state of structures only periodically. The reasons for the delays are financial, lack of man power, and inaccessible structures.

Damages induced in existing and new structures could be in the material or structural properties of the structure, thereby deteriorating serviceability. Concrete and steel cracks, concrete voids, delamination, corrosion, and bolt loosening are common material damages witnessed in the structures. Meanwhile, deflection, changes in stiffness, dampness, and mass are structural 
properties that define the state of a structure's health and are altered due to the aforementioned damages. Though material defects will cause changes in structural properties, that is not always the case. Minor defects encourage further deterioration but can be detected and cured beforehand. Damages in the structures, however, are unpreventable during their lifespan. Therefore, to ensure the safety of a structure and the users, it is vital to assess the health of a structure over time and after major events (earthquakes, hurricanes, accidents). The acknowledged damages may propagate to a severe level if not detected at early stages and may cause accidents. Immediate inspection is delayed by various factors such as the severe climate, location, light conditions, finances etc., [4].

\subsection{Problem Definition}

Worldwide, the layout of structures is expansive and the need for new structures is increasing day by day. Existing structures cannot be rebuilt but instead can be safeguarded by damage detection and rectifying the detected damages. Thus, structural health monitoring (SHM) is a process adopted by engineers to diagnose structures for damages in material and changes in structural properties. The SHM process can be summarized into four phases [5]:

1. Damage detection

2. Damage localization

3. Damage quantification

4. Structure safety or service life analysis 
In the past decades, various damage detection methods have been developed such as manual inspection, contact sensor-based damage detection, and vision-based damage detection. Every method has its limitations and no single method can overcome all limitations. Moreover, one method might suit the conditions of a particular structure and type of damage, whereas another method might give better results for another type of structure and damage. Further, as mentioned earlier, structure location and availability of man power also limits the use of some manual inspection methods. Therefore, more options will lead to timely detections and assessments. The adaptability of a method depends on various factors such as location, environmental conditions, type of damage etc.

Manual inspection is the oldest and most widely adopted method to date and is time, money, and labour intensive. For structures located remotely, however, damage detection becomes even more complex since periodic checkups of structures is more intensive in terms of time and finances. Furthermore, this method provides a subjective detail account of damages since the details vary from individual to individual, and depend on the opinion and experience of the inspector [5]. Additionally, damages like cracks, delamination, corrosion etc., can be reported manually, but voids or internal damages cannot always be inspected manually. To overcome limitations of manual inspection methods, sensor-based and vision-based methods were developed. Sensorbased methods provide promising damage detection but may not be always applicable as discussed further in the literature review. As a result, vision-based methods were developed as alternatives to sensor-based methods. Moreover, in the case of loosened bolt detection, not many computer vision-based methods are available. Developing automated methods eliminates human dependency, is cost effective, as well as fast tracks structure inspection. 


\subsection{Research Objective}

The research objectives of the study are:

1. To develop an automated vision-based loosened bolt detection method

2. To improve the limitations of existing vision-based loosened bolt detection methods

3. To provide a parametric study of the proposed method and study the limitations:

a. Vertical angle of the bolt from camera lens

b. Horizontal angle applicability

c. Loosened length study

\subsection{Methodology}

The proposed model methodology is based on two algorithms - the Viola-Jones algorithm for detection of bolts in the image and support vector machines (SVMs) for classification of loosened and tight bolts. The cascade bolt detector (CBD) is based on the Viola-Jones algorithm and is trained for detecting bolts. The trained CBD detects the bolts in the input test images and crops the bolts individually. The SVM is also trained for loosened and tightened bolt classification. The images cropped by the CBD are fed to the trained SVM for classification of loosened and tight bolts. The detailed working methodology of aforementioned methods are discussed in chapter 3 of the thesis. 


\subsection{Thesis Organization}

The thesis consists of 5 chapters as follows:

- Chapter 1 introduces the research through problem definition, objectives and methodology followed to achieve the objectives of research.

- Chapter 2 provides the information about the various damage detection methods developed to the date. The review of limitations of the existing methods which lead to the proposed methodology.

- Chapter 3 provides the detailed working of developed vision based loosened bolt detection algorithm.

- Chapter 4 provides the parametric study and performance of developed algorithm with limitations.

- Chapter 5 summarizes the outcome of the research. The scope of future work is also covered in this chapter. 


\section{Chapter 2. Literature Review}

In this Chapter, a literature review of the previously proposed contact sensor and vision-based methods is concluded. Firstly, a review of the contact sensor-based methods is listed, followed by the pros and cons of contact sensor-based methods, leading to the development of vision-based methodologies.

\subsection{Background}

In civil structures, damages can be classified into structural and material damages. These damages will force a change in the modal properties of structures such as stiffness, mass, and dampness, but the material damages may not always have an impact on the structural properties. Despite that, either type of damage needs to be identified and rectified to prevent further deterioration of the structure. There is no single solution to detect all types of damages. Both the methods - visionbased and sensor-based are handy at times.

The most common damage in concrete structures are cracks. They might be micro cracks or macro cracks. Micro cracks are the outcome of poor workmanship, low quality material, and inadequate mixing, as well as the environment during the concrete pouring. Though these cracks have a minor impact on natural frequencies and mode shapes, but they can prompt corrosion of reinforced steel [6]. Macro cracks, on the other hand, alter the natural frequencies and mode shapes of the members. In steel structures, the witnessed damages include member and bolt corrosion, cracks in the steel members and joints, bolt loosening at joints, and imperfections in welded joints. All of these damages cause a shift in the natural frequencies and mode shapes and can lead to failure of the 
structures if not repaired or replaced. Figure 2.1 shows some common types of damages in the structures [7-10].

The damages might not affect the performance of a structure negatively but they have to be monitored to ensure the safety of the structure. As mentioned in Chapter 1, the SHM is a combination of damage detection and the ensuring of the safe performance life of the structure. To ensure the state of health, civil structures are monitored periodically and examined for changes in the structural behavior. However, manual inspection of infrastructure is the most widely adopted method. The limitations of the manual detection methods listed in Chapter 1 lead to the contact sensors based methods [11].
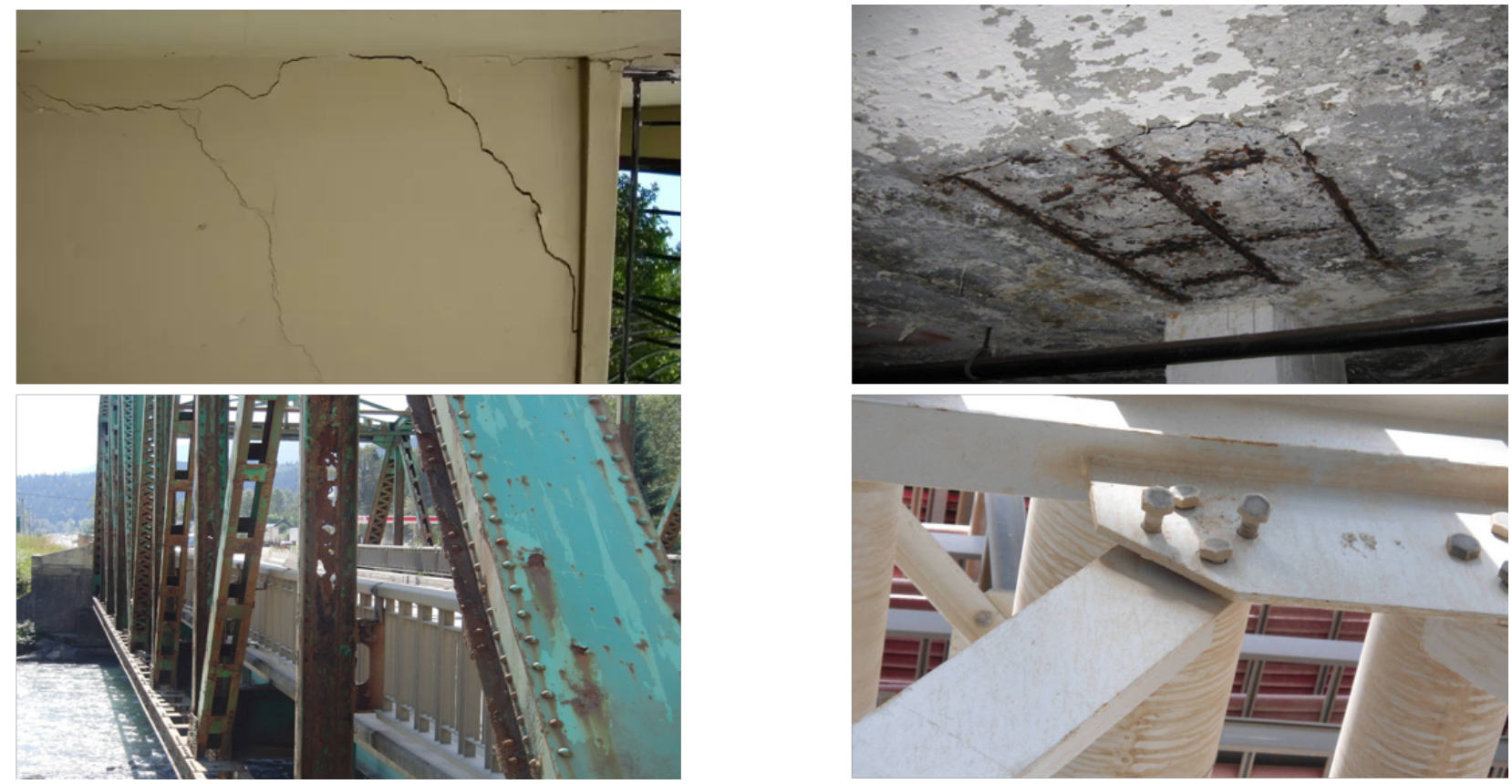

Figure 2.1. Types of defects in civil structures

(a) cracks [7], (b) delamination [8], (c) corrosion [9], (d) loosened bolts [10] 


\subsection{Contact sensor-based methods}

A contact sensor collects the data through a set of sensors and is processed by the central processing unit for damage information (Figure 2.2). Structural member's damages alter the physical properties (stiffness, mass, and damping) resulting in changes in frequencies and mode shapes. Further, these methods can be classified into the data/response-based and modal-based methods. If the numerical model of the structure is available for damage identification, the method falls under model-based; if the testing is based on the frequency responses of the structure, the method is response/data-based methods [12]. Contact sensor-based methods detect the damage through natural frequencies and mode shapes of the structures [13]. To date, many contact sensorsbased methods have been developed for the monitoring and damage detection of structures [1418]. From the available literature, the contact sensor-based methods comprise of vibrations, wavelets, ultrasonic methods, model updating methods etc. Researchers have applied theories to existing methods to improve the sensitivity and applicability of methods, some of which are listed below.

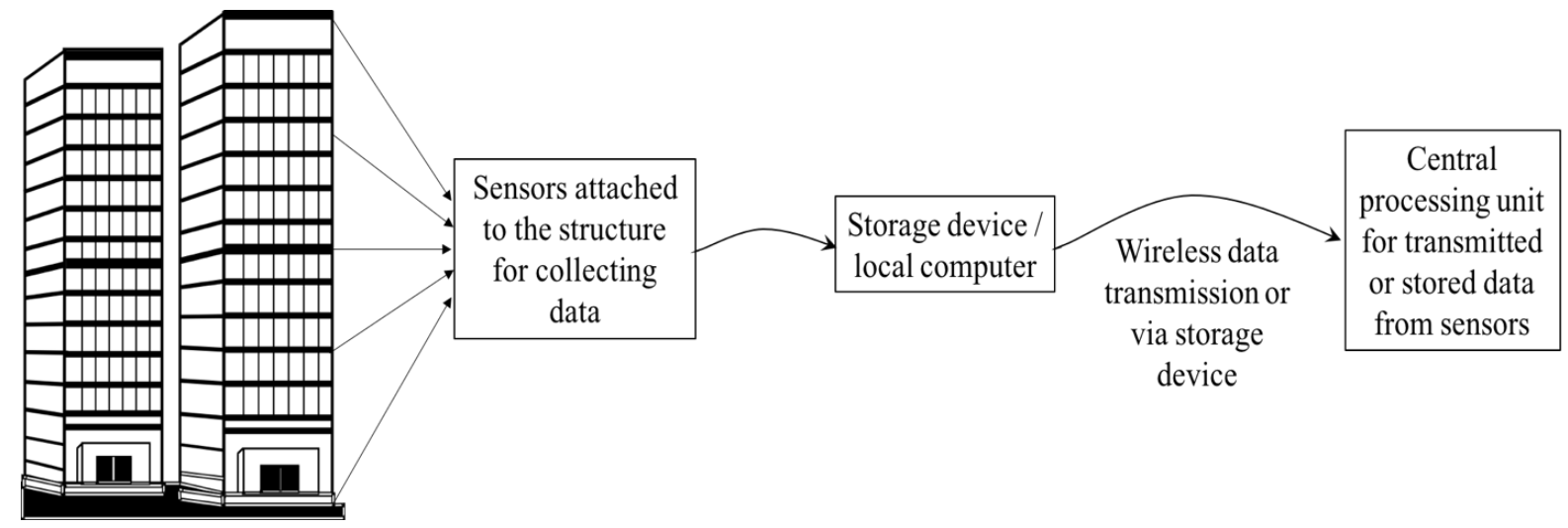

Figure 2.2. A schematic diagram of sensor based damage detection method. 


\subsubsection{Contact sensor damage detection in concrete structures}

Data is collected from the structure as frequency responses, wavelets etc. The collected data is processed and compared to numerical calculations or previously collected data for detecting damages. Any variations in the responses leads to the detection and further damage quantification. Sensor-based methods have been extensively researched; Lead Zirconate Titanate/piezoelectric (PZT) sensors are the most commonly used patches, and can be used as an actuator and receiver at the same time. Moreover, PZT has a higher sensitivity and a wider variety of shapes and sizes. Soh et. al. [19] used PZT sensors on a RC beam to test the sensitivity and effectiveness of the sensors. The bond between the PZT sensor and the structure also affected the quality of the data collected. Their study concluded that PZT sensors are sensitive to cracks at the early stage, and thus provide early stage warning. Moreover, the sensitivity of the sensor depends on the distance from the crack; with an increase in distance, sensitivity drops and cracks might not be detected. Wang et. al. [20] and Song et. al. [21] tested PZT's for reinforcement debonding and cracks in concrete bent caps. To measure the extent of the damage, PZT sensors were attached prior to casting. The sensors were able to detect and track the growth of the cracks with time. Long span bridges continuously undergo daily heavy cyclic loading and crack propagation is a major concern in bridges. Energy drops between the actuator and the sensor indicate crack development.

Mekjavic et. al. [22] proposed a damage detection method using high natural frequency response. The vibrations were measured before and after the damages and were induced by the electromagnetic actuator, whereas the piezoelectric accelerometers collected the responses. The method was validated by comparing the outputs with theoretical equations. The change in test beam stiffness due to cracks resulted in alteration of the measured frequencies. The method was 
effective in localizing and quantifying the cracks without prior knowledge of mode shapes. Natural frequencies are quite simplified and easy to measure for structures. In another study, Kim et. al. [23] analyzed the beam using frequency and mode shape changes for crack detection, localization, and quantification. The purpose of the study was to evaluate the accuracies of both methods and results were validated using finite element analysis of the beam. The method detected the cracks and crack localization was within $4 \%$ error, which is very promising for heavy civil structures. For damage identification, the data has to be processed. Park et. al. [24] proposed a real-time multiple crack detection method by measuring the impedance of PZT sensors. Two frequency modes were tested: lateral $(>20 \mathrm{kHz})$ and thickness $(>1 \mathrm{MHz})$. The finite element model of the test specimen assured that both modes detected the crack, but the lateral mode was found to be more sensitive to multiple crack detection since cracks are at different distance from the sensor.

Low vibration frequencies are not always sensitive to local damages or small damages in the structure. Another damage detection approach is finite element model (FEM) which updates the structure and visualizes changes in the dynamic properties (mode shapes, natural frequencies) [25]. The FEM-based method can be described in three steps [25-26]:

1. The finite element model of the structure is developed with intact dynamic properties as close to the real structure as possible

2. The model is updated to replicate the damaged state of structure

3. To reduce the disparity between model and the experiment structure, the changes in the physical parameters of the finite element model refer to the damages induced 
Teughels et. al. [25] performed an experiment on the Z24 concrete bridge in Switzerland. The eigen-frequicies and mode shapes were extracted using accelerometers over the span of the bridge. The damage in the structure was identified in terms of stiffness reduction in the bridge. Moaveni et. al. [26] used the first three longitudinal mode shapes and natural frequencies of a six story structure to model and update the model for validity purposes.

\subsubsection{Contact sensor damage detection in steel structures}

Civil structures also include steel structures. Unlike concrete structures, steel structures can get corroded and connections can get loose due to the loosening of bolts or cracking of the welds, whereas cracks in steel can cause structural damages as well. The base concept of detecting damage in steel structures is similar to that of concrete structures. Researchers have optimized the existing natural frequency-based, impedance-based, wavelet-based etc., methodologies to detect and localize damages. Park et. al. [27] experimented the steel plates by measuring PZT sensor impedance for detecting cracks. Changes in the structure such as cracks in the steel plate can be represented by variations in electrical impedance of a PZT sensor. The closer the damage is to the PZT sensor, the more is the accuracy. Additionally, the experiment was built on previously developed methods as it used a higher frequency $>1 \mathrm{MHz}$ than the conventional range $>20 \mathrm{kHz}$. Doyle et. al. [28] experimented with acousto-elastic and magneto-mechanical impedance methods for loosened bolt detection. Piezoelectric sensors and magneto elastic active sensors measured wave variations due to stress changes. In the acousto-elastic method, the damage sensitive feature for loosened and tight bolts was the wave phase difference, found to be very effective in the experiment. The magneto-mechanical impedance method used impedance peak variation to 
differentiate the status of the bolted joints. Although the acousto-elastic method performed better than the latter, the sensitivity of the sensors to bolted joint stress changes was very low.

Chen et. al. [29] used PZT sensors to measure electro-magnetic impedance steel and aluminum. Arguably because PZT sensors can simultaneously act as an actuator and a sensor. The PZT's were found to be sensitive to the bolt distance regardless of the material and frequency band as in the case of concrete structures. Sevillano et. al. [30] introduced a combination of electro-mechanical impedance (EMI) and guided waves to amalgamate the features of both methods. Damage is detected by the electro-mechanical power dissipation generated from EMI and the fast Fourier transform (FFT) and inverse fast Fourier transform of the guided waves. The method was tested on a variety of structures such as beams, fiber reinforced polymer materials, and bolt connections. However, in the bolt connection scenario, loosened bolts cannot be localized. Furthermore, single or multiple damages cannot be distinguished [30]. Huda et. al. [31] developed a loosened bolt detection method using accelerometers and non-contact laser excitation. This was used to generate uniform repetitive excitation and high frequency vibrations during sampling, giving better outcomes for natural frequency and mode shape changes. Weng et. al. [32] and Frtetzen et. al. [33] experimented with FE model updating for the detection of the loosened bolts and cracks in steel plates, respectively. Their experiments detected damages in terms of reduction of stiffness. The resulting accuracy largely depends on the intact model state of the structure. Discrepancies in the structure modelling will lead to the false detections.

Hence, contact sensor-based methods overcome the limitations of manual inspection. However, there are limitations to contact sensor methods as well. Firstly, in the above mentioned methods, the sensitivity of the sensor depends on the distance from the damage. If the sensor is close to 
induced damage, the damaged is detected and localized; however, if the damage is relatively far, the alarm can be doubtful. Thus alarms are confirmed by physical verification. Furthermore, to evaluate large structures such as buildings or complex structures such as bridges, the required number of sensors will be relatively too large. Secondly, in the FEA model updating methods, results are dominated by modelling accuracy. The models have to be optimized but for large complex structures, and developing an accurate model is close to impossible. If a modelling disparity occurs, the damage detection results cannot be trusted. Thirdly, in the above methods, the collected data reliability of the sensors is questionable. The contact sensors are uncertain and the collected data is vulnerable to environmental effects, as the data from sensors is affected by humidity and temperature [34-35]. As temperature increases, the natural frequencies of the structures decreases, which is also the case of an increase in humidity [34]. Many temperature compensation methods were introduced to counter the effect of temperature on natural frequencies but were not effective, since temperature is not uniform throughout the structure. Moreover, natural frequencies of concrete structures are more sensitive to temperature than those of steel, which can vary by $0.15 \%$ per degree change in temperature [34]. As a result, the collected data may give false alarms and have to be validated manually every time. Meanwhile, attaching sensors to the structures can be a challenging task due to weather and the geometry of the structure [36].

\subsection{Vision-based damage detection methods}

Therefore, vision-based detection methods are the practical alternative to overcome the limitations of sensor-based damage detection methods. Vision-based methods are relatively new in SHM in comparison to sensor-based methods, but have enormous opportunities within the field. There are many advantages to vision-based methods such as a very simplified process, universality, along 
with low cost of monitoring. Vision-based damage detection is composed of an image capturing device (camera), a computer, and a processing algorithm for detecting damages in the images (Figure 2.3). Researchers have developed techniques for detecting damages such as cracks, delamination, deflections, corrosion, bolt loosening, etc., which are further briefed in this chapter. Moreover, the methods reviewed in the literature use cameras instead of laser scanners for damage detection. This is because laser scanners are expensive and bigger in size than cameras, and secondly, because the external power requirement is significant for lasers [37]. Hence, most studies in the literature have focused on digital cameras.
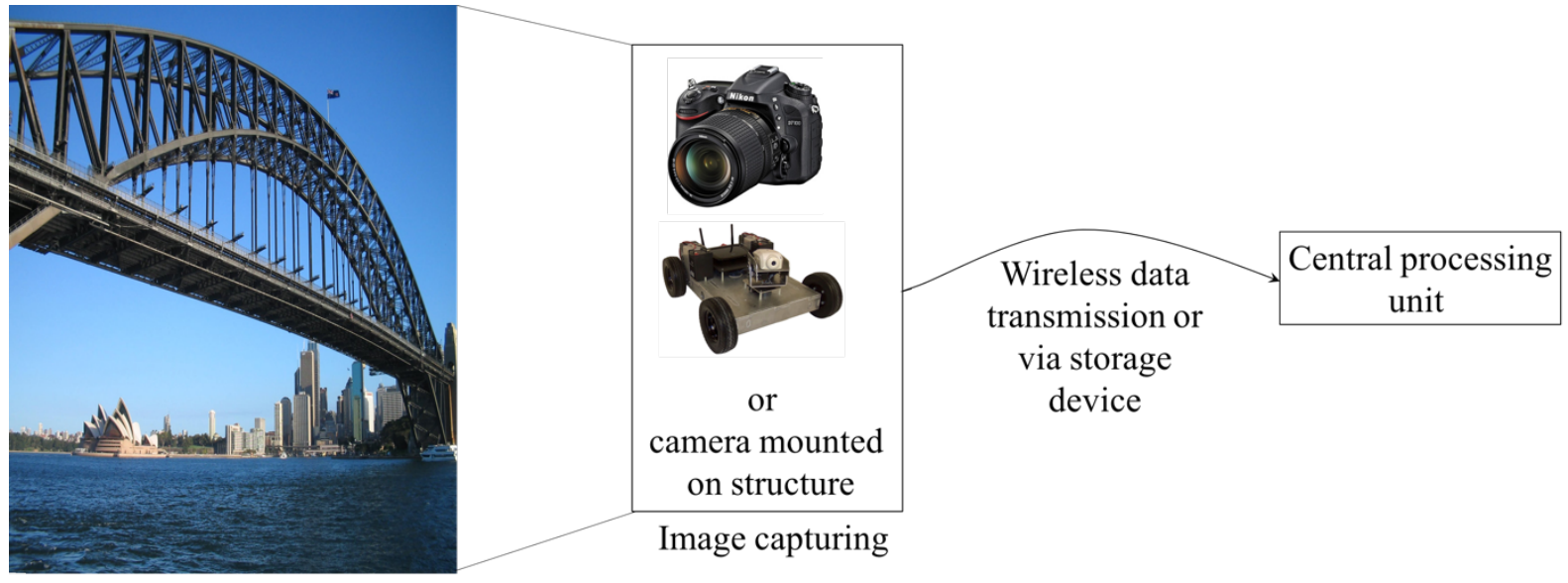

Structure to be examined

Figure 2.3. A basic process of image processing algorithm.

2.3.1 Crack detection by vision-based methods

Cracks are the most commonly observed damages to structures. The distinction between crack and no crack surface in the image is the contrast difference. Pixels in the crack image space will be 
darker than in the no crack image space despite the material (concrete, pavement, etc.), which is the principle for detecting cracks in vision-based crack detection. Images will include noise as well, such as, shadows, blurs etc., and thus, have to be taken care or else can provide the wrong output. Researchers have proposed and optimized a variety of image-based crack detection methods for various conditions and structures.

Yu et. al. [38] proposed a semi-automatic vision-based crack detection method for tunnels and subways etc. The motivation behind the research was to eliminate subjective manual inspection and propose a cost effective accurate crack detection method. A mobile robot with a mounted camera and a light illuminator was used to capture and store images. An algorithm processed the images, detected cracks, and discarded non-cracks due to noise and illuminations. This method quantified cracks with width, length, and direction of the crack using Sobel and Laplacian operators. The algorithm was tested at lab scale with an accuracy of about $85 \%$. For evaluation crack width and length, Yamaguchi et. al. [39] proposed the percolation method for segregating and processing cracks in image space. Chen et. al. [40] proposed a method for detecting crack continuity in series of images since the complete structure cannot be captured in a single image. The crack width and length was measured as well. Meanwhile, Huang et. al. [41] proposed a pavement crack detection method with the camera mounted on a pavement inspection vehicle. Instead of detecting cracks in a full size image, a grid of $8 \times 8$ pixels was evaluated. Detecting the potential cracks out of the false detections generated due to noise connectivity of dark pixels was examined. Although these methods were performed under controlled conditions, the results were promising for future research to develop and improve on the existing ideas at the field level. 
Vision-based methods research is focused on eliminating existing limitations as well as the application of methods to real world problems. One of these is the monitoring the health of structures periodically, and the other is post disaster evaluation of structures during earthquakes and manual disasters. The latter scenario involves two challenges: (1) real time damage evaluation, and (2) the accuracy of detection is the paramount requirement for safeguarding the building for human inspection or use. Torok et. al. [42] developed a post disaster 3-dimensional damage detection method for buildings. The basic concept is same as in previous methods with the camera mounted on a manually controlled robotic vehicle. The concern with vision-based crack detection methods is the state of images captured for damage detection. Varying light intensity, shadows, blurred images etc., can all reduce the performance accuracy of methods. To overcome these limitations in crack detection, Cha et. al. [43] developed a crack detection method based on convolution neural networks (CNN). The method was validated on low light to bright images and blurred images and produced an almost $97 \%$ accurate crack detection. The testing was performed on $40 \mathrm{~K}$ images with a $256 \times 256$ resolution. The method was compared with Canny and Sobel edge detection methods, the results showing that deep learning CNN performed better than both the well known damage detection methods. Since larger images were cropped down to a $256 \times 256$ resolution, the images with cracks at the edges were disregarded because they might lead to the false CNN training.

\subsubsection{Corrosion detection by vision-based methods}

Defects visible on the surface of the structures can be detected with vision-based methods. Corrosion - occurring on steel structures such as bridges, pipelines, sections etc., is one such damage. Various methods have been developed to detect corrosion in bridges, boilers, pipelines 
[44-46]. Chen et. al. [44] introduced automated rust detection methods in steel surfaces based on FFT and SVM. Instead of using RGB, LAB colour space was used for the image processing; moreover, only the blue-yellow $\left({ }^{*} \mathrm{~B}\right)$ component of LAB was used for the rust detection. The original image was compared for similarity with the FFT and inverse FFT processed image. Further, images with difference in colour space were used to train the SVM for rust defect detection and no defect images. Pascual et. al. [45] evaluated the patch initially; once the patch is doubted as corroded, the pixel in the patch is evaluated to label the region as corroded.

\subsubsection{Displacement by vision-based methods}

Image processing technique advancements in the SHM field have led to the vision-based methods for measuring the displacement in structures as well. Various vision-based displacement monitoring systems have been applied in the last decade. Uhl et. al. [47] used unloaded and loaded specimen images pixel correlation to calculate deflection. Markers were used as match points in the images and were independent of deformation of the specimen due to applied load. The normalized cross correlation coefficient was used to get the deflection readings after application of the load. The method was tested at lab scale and readings were compared with the laser sensor. The developed method discrepancy was less than $0.5 \%$. Ye et. al. [48] introduced a real time, long range vision- based displacement measurement system. The method was applicable up to the distance of $1000 \mathrm{~m}$ 's. The main idea of the system is to measure the coordinate difference in a series of images of a LED lamp attached to the bridge for displacement measurement. Same as in the above mentioned method, cross correlation was used to find the similarities between the patterns being tracked. The method was validated at lab scale by comparing the results of visionbased, vibration tests, and laser transducers. The test was performed in the field, and tested "Tsing 
ma" bridge in Hong Kong for midspan displacement. The method provided satisfactory real time results from a range of $1 \mathrm{~km}$.

Grano et. al. [49] developed a displacement measurement method based on a Kinect sensor using depth images. Another prominent study by Cha et. al. [50] introduced phase-based optical flow for displacement measurement and unscented Kalman filter for reducing measurement noise. Here, state space equations damage was identified using system stiffness and damping. The most important part - excitation input knowledge was not required for the test. The method was validated on the intact and damaged cantilever beam. The method offers an opportunity for future development of more complex structures such as high-rise buildings.

\subsubsection{Loosened bolt detection by vision-based methods}

Civil structure comprises of various members and often, in steel structures, the structural members have bolt connections or welds. Therefore, along with from cracks in steel, corrosion, and buckling, another kind of defect in the structures is bolt loosening. In a study conducted by the Korea Expressway Corporation, 33\% bridges were found to have bolt defects and the major cause of bolt defects was bolt loosening [51]. For detecting a loosened bolt, the contact sensor-based methods such as impact hammer and torque ranches are available. However, bolted connections are complex and connecting sensors and performing a periodic check are not handy tasks. Visionbased methods are the alternative to contact sensor methods. Park et. al. [51] developed an angular loosened bolt detection method using Hough's transform. This method is limited to only angular bolts, and moreover, the images with top view of the bolt work with this method. The results were promising, however, and this study provided an opportunity for future development of loosened bolt detection. This method was further applied to the detection of loosened bolts in a moving 
turbine [52]. In another study, Cha et. al. [53] introduced a method based on Hough's transform and SVM. The bolt features are extracted from individual cropped bolt images and the SVM is trained to classify loosened and tightened bolts based on the extracted bolt features. Again, this method was limited to bolts with ranch holes only.

To date, very limited vision-based methods have been developed. Bolts are a significant member of the steel structures. The limitations and limited methods available for loosened bolts is this study's motivation to develop a more generic method based on existing studies with promising results [51-53]. The main motive behind this research is to develop an automated method applicable to different bolt heads. 


\section{Chapter 3 - Methodology of vision based damage detection}

\section{for bolts}

The following chapter introduces an overview of the vision-based loosened bolt damage detection method based on the Viola Jones algorithm and SVM. The algorithms involved are summarized, including the feature extraction and working methodology of algorithms described.

\subsection{Overview of the vision based loosened bolt detection method}

The developed method is a combination of two methods; cascade bolt detector (CBD) and SVM. The CBD is based on the Viola-Jones algorithm for detection of the bolts in test images. The input image contains bolts and other structural components. To classify the loosened and tight bolts, the bolts have to be isolated from other components in the image. The CBD is trained to detect and crop the isolated bolt images from input test images provided by the camera for classification. The original classifier is trained for detecting human faces, nose, eyes. However, for the detection of any other object, the classifier has to be trained for a detection of a particular object. Therefore, $\mathrm{CBD}$ is trained for detecting the bolts in the developed method. Figure 3.1 presents an overview of the proposed method. CBD is trained on the extracted features of a classified dataset of images through Adaboost meta-algorithm [54]. The cropped bolt images are fed to SVM for classification into loosened and tight bolts.

For the classification of loosened and tight bolts, the SVM is trained on another image dataset, as shown in Figure 3.1. The cropped bolt CBD images are fed into the trained SVM for classification based on the extracted feature as proposed by Cha et. al. [53]. The modifications compared to 
existing method are explained in section 3.4.2 of this Chapter. Since the method uses two different algorithms, two different image datasets are used for training. The training images are taken with varying orientations, light intensities, distances, and background. Moreover, the training needs to be done only once for both the methods and can be used for the detection and classification of similar bolt shapes.

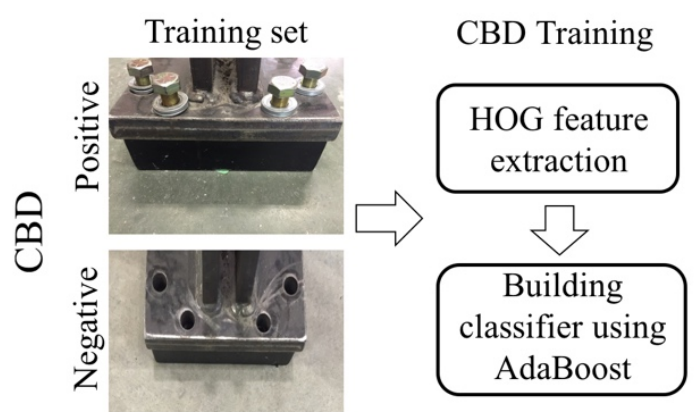

(a) CBD training

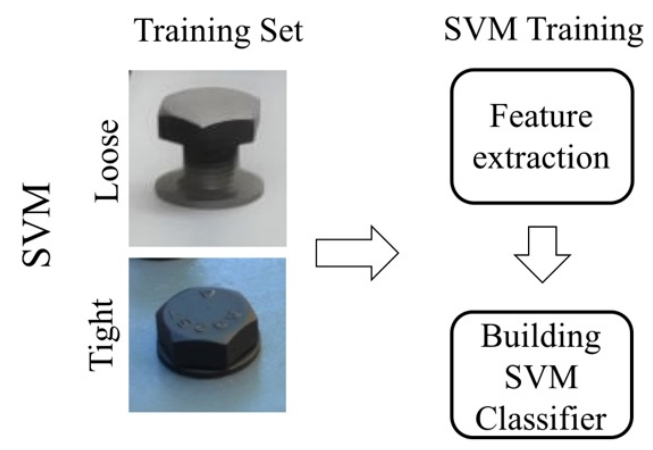

(c) SVM training
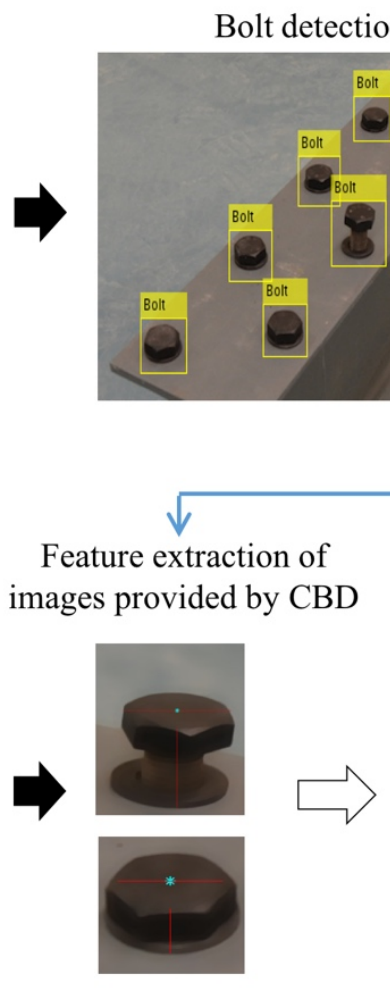

(d) SVM testing
Cropped bolt images

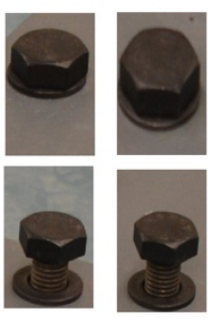

(b) CBD testing

Classification

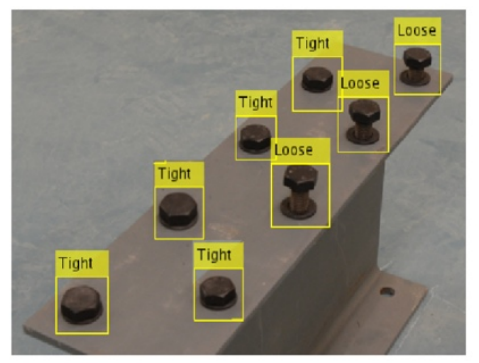

Figure 3.1. Overview of the method.

\subsection{Methodology}

As stated, the proposed method performs the recognition of bolts using a CBD and the classification of loosened and tight localized bolts using an SVM. This section summarizes these 
methods; more detailed explanations of these methods can be found in Viola et. al. [54] and Gunn et. al. [55].

\subsection{Cascade bolt detector (CBD)}

Viola-Jones is the base algorithm for a CBD. The original cascade object detector was used for detecting human faces based on a specific set of features. Also, the default classifier is trained for human faces. To detect the bolts in test images, a CBD is trained using specific features of the classified dataset. The CBD can be trained with Haar like, Histogram of oriented gradients (HOG), or Local binary patterns (LBP) features to detect the intended object. These three feature descriptors extract valuable information from the object image regions for detection and recognition purposes. The default cascade classifier for detecting human faces is trained using Haar like features. Feature descriptors are described below.

\subsubsection{Haar like features}

One basic feature of an image is intensity variation. Image intensity is different for different objects. For example, when visualizing a car, the image intensity of the tire region, body, windows, lights, etc., will differ from each other. The Haar features descriptors are based on the intensity difference between the regions of the image or simply the intensity-based descriptors. Much like the human face has various features such as, eyes, nose, cheeks, each face feature will have a different pixel value as the image intensity varies since eyes are darker than cheeks and nose is brighter than cheeks [56]. Viola Jones used Haar features for robust face detection using a cascade object detector. Further, Haar features are categorized into two, three, and four rectangle features, as shown in Figure 3.2. The white region pixel sum is subtracted from the grey region pixel sum, 
where the size of the rectangular region is the same for white and gray regions [54]. For robust calculation of the pixel sum differences, Viola et. al. [54] inherited the integral image calculations in their study. Moreover, rectangular features are sensitive to edges and changes in other image features. To generate a set of Haar features for an image, a scanning window sized $24 \times 24$ scans the image and features are extracted. The scanning window size increments by 1.25 times the previous size until it goes to the biggest possible size for an image [54].

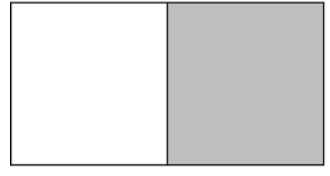

(a)

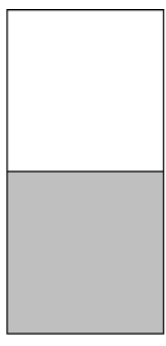

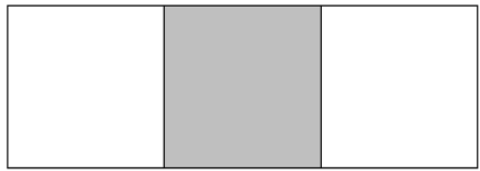

(b)

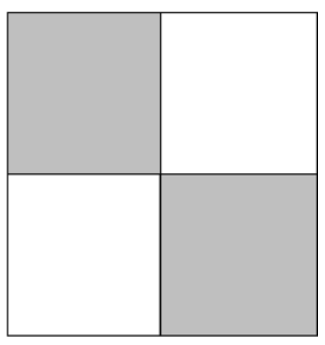

(c)

Figure 3.2. Haar features (a) two rectangle feature (b) three rectangle feature (c) four rectangle feature.

\subsubsection{Local binary Patterns (LBP) features}

LBP is another type of descriptor used in image processing for classification. This feature is based on the texture variations in image. When visualizing a concrete wall with cracks, the texture of wall is different from the crack or window etc. The feature can be briefly defined from the name “LBP”, meaning converting the gray scale image into binary patterns [57]. The image is divided into smaller regions, where every pixel is evaluated by its eight neighboring pixels. If the value of a central pixel is higher than its neighbor, the new value of the neighbor will be 1 , otherwise 0 , 
where the central pixel value is threshold, and thus, the grayscale values are converted to binary patterns and are added to give the value of the central pixel [58].

\subsubsection{Histogram of oriented gradients (HOG) features}

The third type of image descriptor in cascade object detector is HOG. The HOG is gradient-based; the features are extracted in terms of the edges in an image and their direction and can define the shape of an object from precise and detailed images [59]. The HOG feature is explained in more detail further in this chapter.

For bolt detection, HOG features were used over the Haar like and LBP. The reasons favoring the HOG features were:

1) Since Haar features are intensity based descriptors, bolt intensity varies with shank and head; due to reflections, intensity also varies a lot. Moreover, the other objects in the image similar to bolt intensity will be classified as bolts

2) LBP features are texture-based features but they work best if combined with some other feature extractor

3) Trial and error method was used for all the feature descriptor with varying input arguments; apparently, HOG features provided the most optimal results because of uniform shape of bolt throughout

4) Computational time was another factor ( $2.6 \mathrm{GHz}$ dual core $\mathrm{CPU})$. Haar features were more time consuming, where as the LBP features were the least time consuming but also gave many false detections as compared to HOG features. Also, computational time between LBP and HOG feature descriptors was not significantly different. 


\subsubsection{Training dataset for CBD}

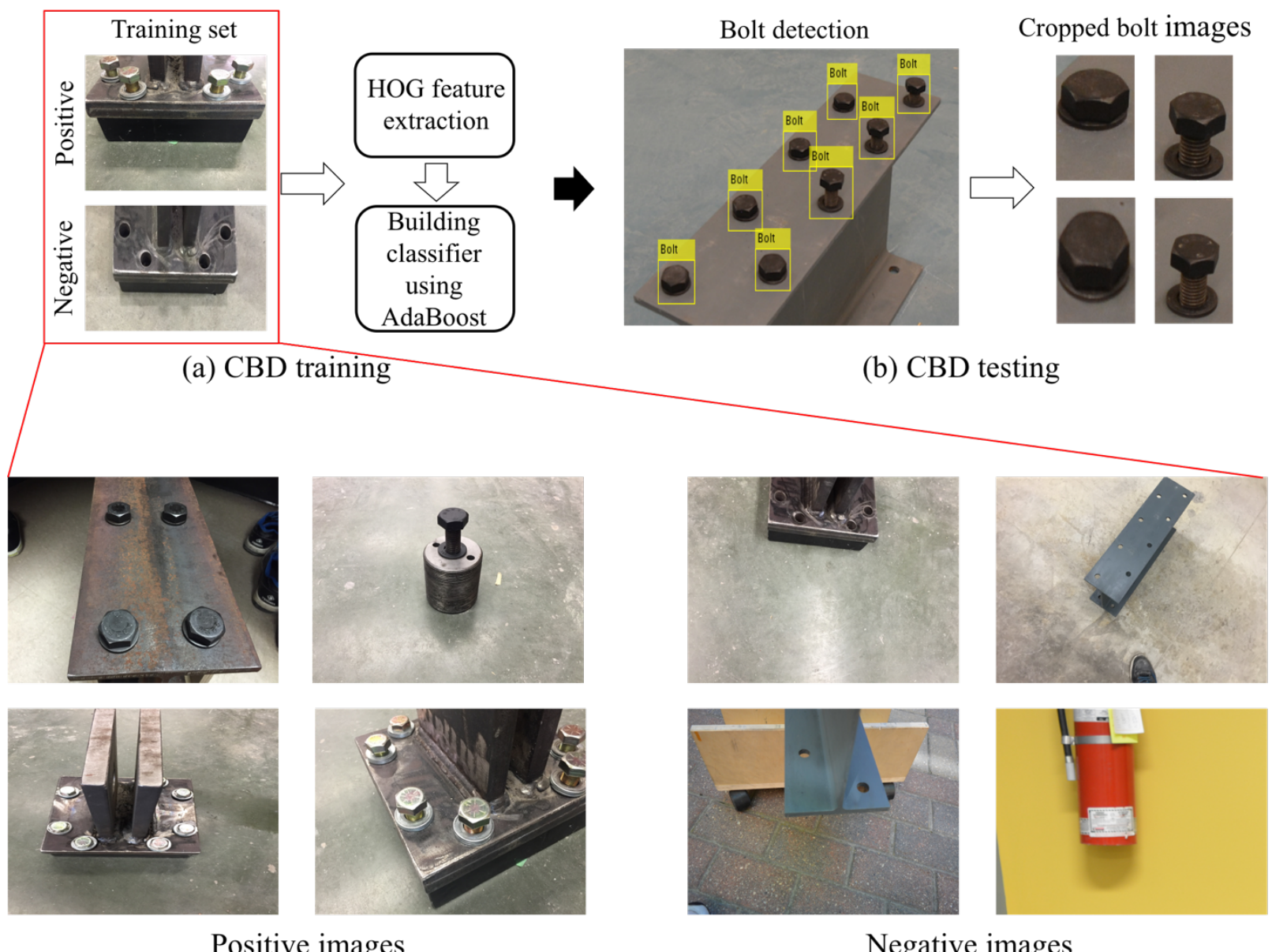

Figure 3.3. Training dataset for CBD, images with bolts are positive dataset; images without bolts are negative dataset.

Thus, CBD is trained using the HOG features of the bolts. The training dataset for CBD comprises of the positive and negative set of the images as shown in Figure 3.3. The positive images dataset contains bolts in the images, regardless of whether they are loose or tight and the set of negative images contains images without bolts such as steel plates. Positive images and negative images 
were taken from varied orientation, distances, and light intensities. However, the negative images also include objects other than the steel plates which might occur in the background. All the images are taken with a digital single lens reflex (DSLR) camera at random distances and angles. The number of images for positive and negative datasets that are used in this study is 141 and 359 , respectfully, at an average resolution of $1024 \times 768$. Negative and positive datasets are used to differentiate the bolts from the background based on the features of the bolts and background according to the classifiers [54].

In a positive dataset, the bolts are interactively defined by bounding boxes or region of interest (ROI). There are open source tools available for defining the objects with the bounding boxes as well. In training, the CBD should focus on the bolt features only rather than other features in the image space, such as background and random objects other than bolts. Also, there may be more than one bolt in the image. As a result, the bolts are always specified as a region of interest (ROI) as shown in figure 7, because the CBD should only be trained on bolt features. If the bolts are not specified by ROI's, the feature descriptor will extract features of the total image space of all positive test images and CBD will be trained for all the objects instead only for the bolts. Alternatively, the individual or cropped bolt images can be used as a positive dataset. For example, in Figure 3.4 the number of positive samples used by the cascade object trainer will be five, not one. The set of negative images consists of steel plates without bolts and random background images of steel I-sections or the objects that might appear in image space. As the algorithm uses a minimum of two negative images corresponding to each positive image, the number of negative images should be greater. Moreover, the negative images once discarded move out of the training 
process and are not used again. The negative dataset has a very deep impression on training, the images should be close to backgrounds of test images for more robust results.

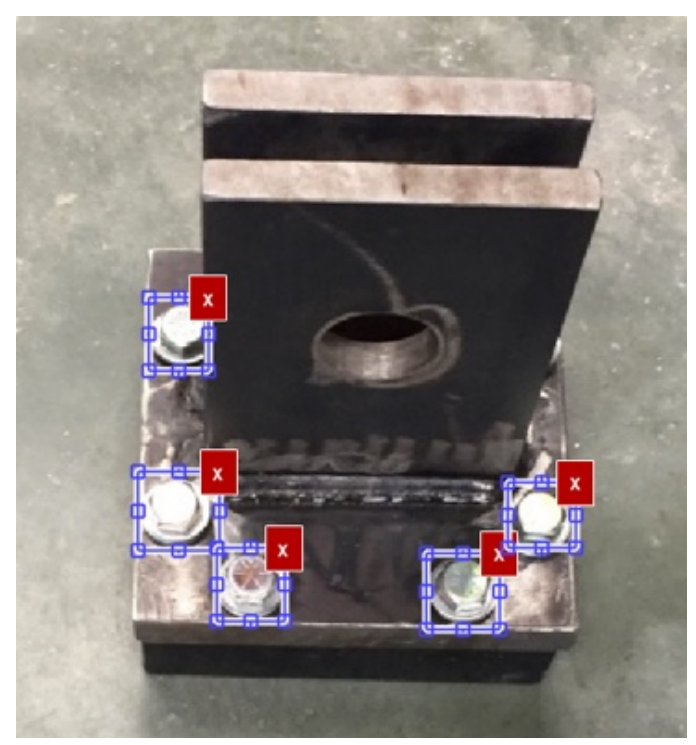

Figure 3.4. ROI's.

3.3.5 HOG feature extraction

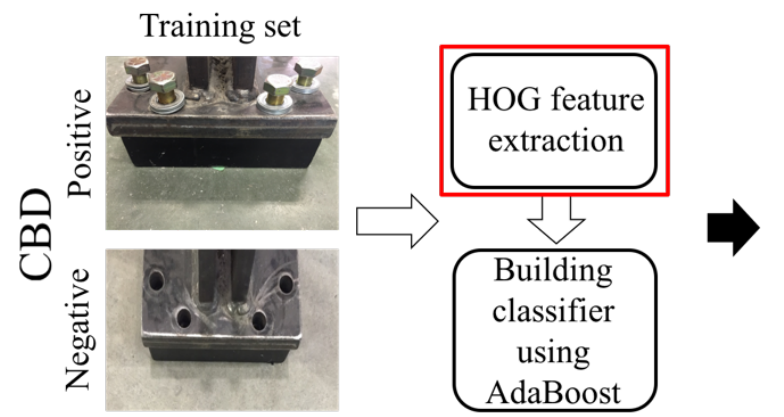

(a) CBD training

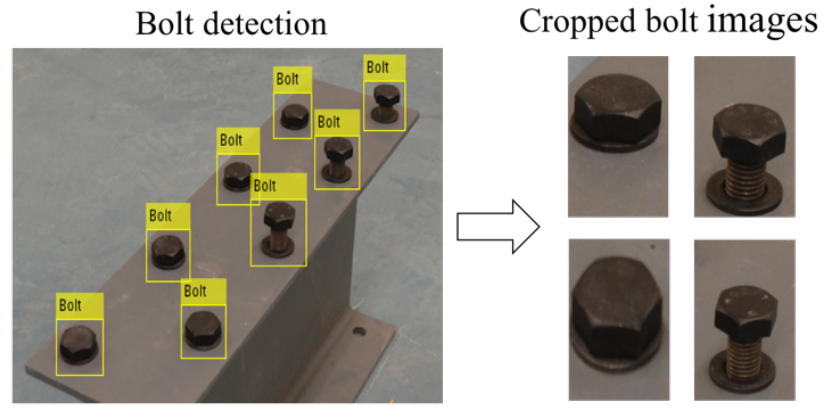

(b) CBD testing

Figure 3.5. This section is focused on HOG feature extraction in CBD training. 
CBD is trained on the HOG features extracted from positive images as mentioned above. The feature extraction process of HOG descriptor is explained in this chapter. Figure 3.6 provides an example of visualization of the HOG features of the bolt. The HOG feature extraction process and is explained further in this part.

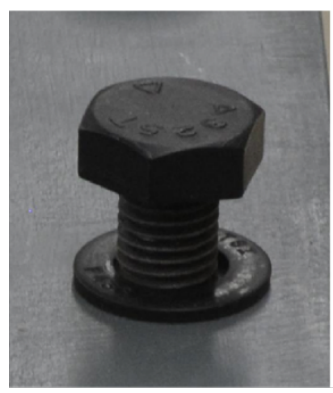

(a) Bolt Image

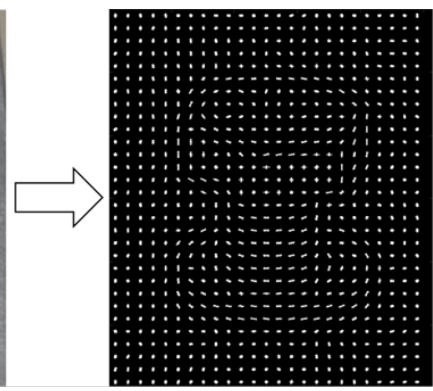

(b) Extracted HOG features

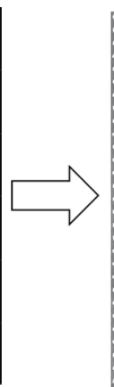$$
\text { . }
$$

.

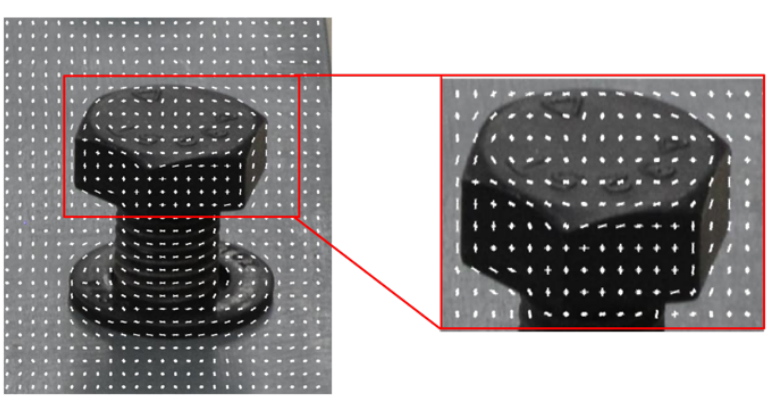

(c) Visualization

(d) Edge Visualization

Figure 3.6. HOG feature visualization of the bolt.

The HOG features for detection were studied by Dalal et. al. [59], the features were used for human detection in the images using SVM. The HOG features were originally designed to divide the input image space into blocks and cells. Where the blocks are formed of $3 \times 3$ cells, and cells are formed by groups of $8 \times 8$ pixels [59]. HOG features can define the object's shape from the edge directions of the object. To overcome the false feature extraction, the blocks are overlapped by $50 \%$ in the horizontal and vertical direction as shown in Figure 3.7. In a cell, each pixel has a certain orientation and the orientation of a cell depends on the summed magnitudes of the pixels [59-61]. At the edge there is intensity variation, stronger the intensity more is the magnitude of the pixel. The gradient orientations and magnitudes of the cells are computed based on the edges located in the pixels as expressed in equations (1) and (2). In the equations, $x$ and $y$ are the values of 
horizontal and vertical gradients for calculating the orientation and magnitude, and $i$ and $j$ represent pixel locations in the image space [60-61].

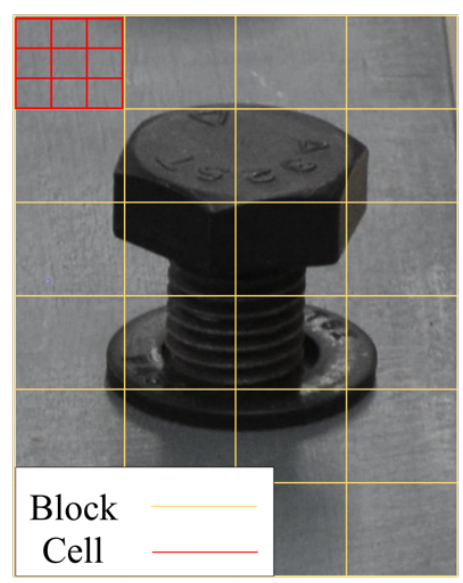

(a)

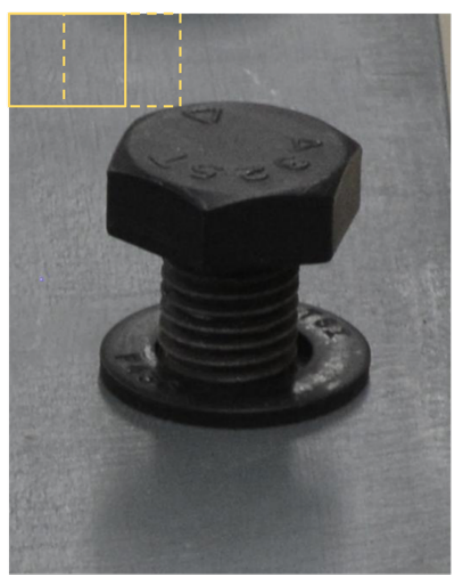

(b)

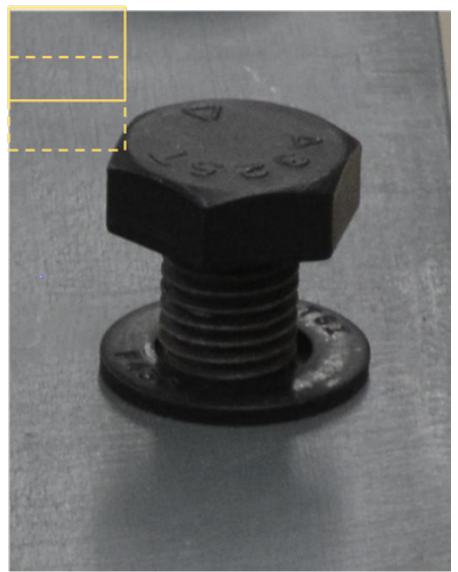

(c)

Figure 3.7. (a) A simplified view of image divided into smaller regions blocks and cells, (b) $50 \%$ block overlapping in horizontal direction, (c) 50\% block overlapping in vertical direction.

$$
\begin{gathered}
\text { Magnitude }=\sqrt{x_{i j}^{2}+y_{i j}^{2}} \\
\text { Orientation }=\arctan \left(\frac{y_{i j}}{x_{i j}}\right)
\end{gathered}
$$

Figure 3.8 explains the HOG feature extraction process on the image patch as shown. The upper part of the image shows pixel values in the cell of $8 \times 8$ pixels. To calculate the gradient, orientation and magnitude of the pixels, $x$ and $y$ directional masks are used. The detailed calculations of gradients, orientation, magnitude, and interpolation of the pixel located at $i=3$ and $j=2$ are shown in Figure 3.8. The $x$ and $y$ gradients are computed by convolving [- $\left.\begin{array}{lll}-1 & 0 & 1\end{array}\right]$ and $\left[\begin{array}{lll}-1 & 0 & 1\end{array}\right]^{\mathrm{T}}$ masks with a pixel and taking the mean values, respectively. For example, when these masks are centered on 
the highlighted pixel, we obtain the gradients of $x_{3,2}$ and $y_{3,2}$ as shown Figure 3.8. The calculation of orientation and magnitude matrices follow equations (1) and (2) after the calculations of the $x$ and $y$ gradients.

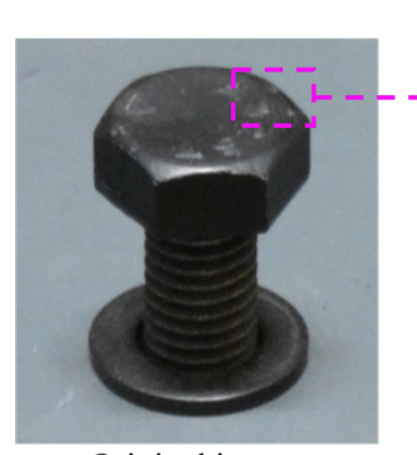

Original image

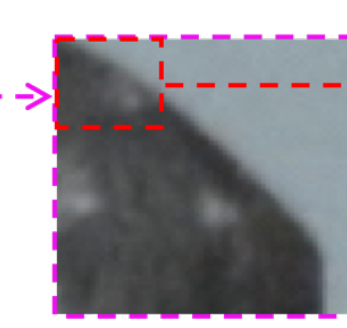

Block (3X3 cells)

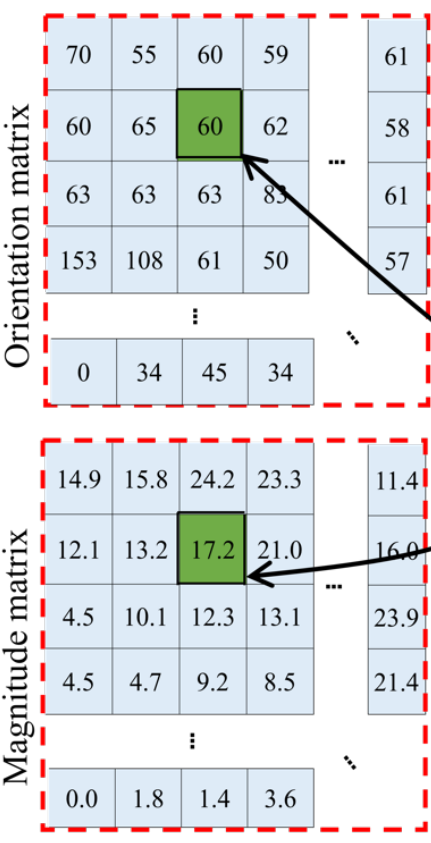

- $x$ and $y$ gradient $x_{3,2}=(96-79) / 2=8.5$

$y_{3,2}=(105-75) / 2=15$

- Orientation and magnitude of gradients

Orientation $_{3,2}=\arctan (15 / 8.5)=60^{\circ}$ Magnitude $_{3,2}=\left(8.5^{2}+15^{2}\right)^{1 / 2}=17.2$

- Interpolation for $40^{\circ}-60^{\circ}$ $\left(70^{\circ}-60^{\circ}\right) / 20^{\circ}$ X $17.2=8.6(50 \%)$

- Interpolation for $60^{\circ}-80^{\circ}$ $\left(60^{\circ}-50^{\circ}\right) / 20^{\circ}$ X $17.2=8.6(50 \%)$

Orientation and magnitude calculation

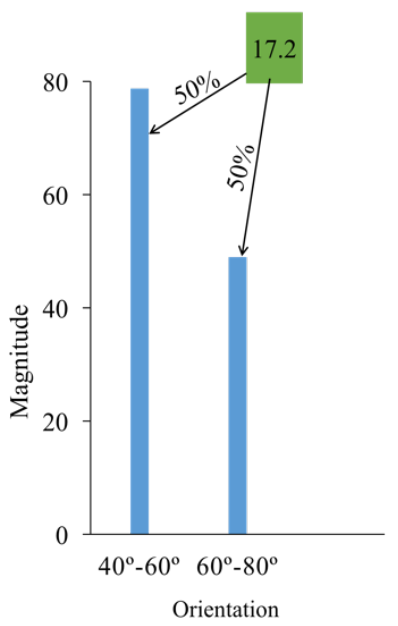

Histogram of pixels

Figure 3.8. Explains the gradient and orientation calculations and interpolation for histogram generation.

The orientations are divided into nine bins of $20^{\circ}$ each from $0^{\circ}$ to $180^{\circ}$, also called unsigned gradients [58]. Dalal et. al. [59] used both signed and unsigned gradients, i.e. $0^{\circ}-360^{\circ}$ and $0^{\circ}-180^{\circ}$ 
respectively, where unsigned gradients outperformed the signed gradients. Thus, 180 is added to all the orientations that do not fall in $0^{\circ}-180^{\circ}$ range. Once the orientations and magnitudes are calculated, the magnitudes of the pixels are bilinearly interpolated to the nearest two bins. In Figure 3.8, the highlighted pixel's magnitude contributes to both of the bins based on the orientation. The magnitudes of the pixels are interpolated into neighboring bins only if the orientations do not fall on the median of the bins. Also in Figure 3.8, the orientation of pixel at $i, j=1$ is $70^{\circ}$, this orientation falls on the median of $60^{\circ}-80^{\circ}$ bin, therefore, the magnitude of this pixel is not interpolated into two bins. To generate the histogram of a cell, the magnitudes of those pixels with similar orientations are summed together. Figure 3.9(c) shows the Histogram of a highlighted cell.

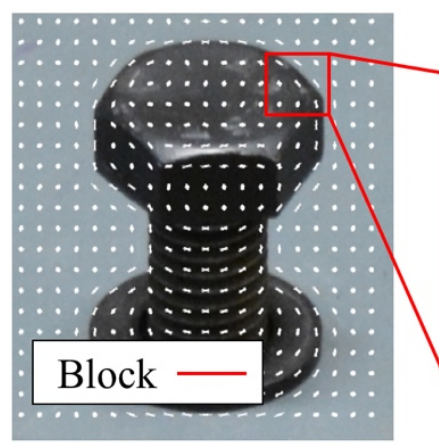

(a)

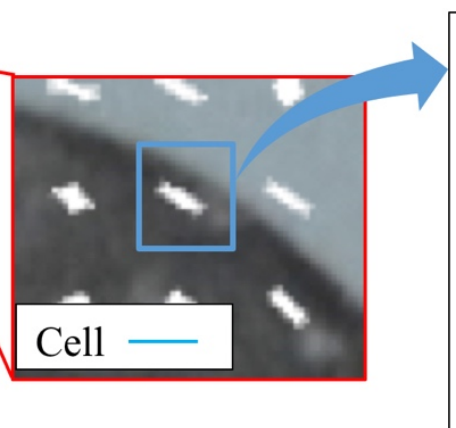

(b)

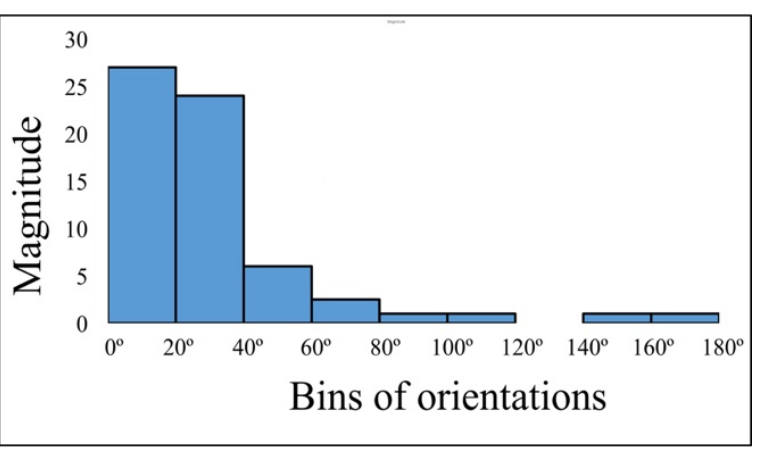

(c)

Figure 3.9. Visualization of the histogram of a cell.

As shown in Figure 3.9 (a) and (b), vectors are oriented in the direction of the edges and represent the dominant direction. The HOG feature vectors of all the cells in a block are concatenated and normalized to remove the effects of contrast variations [59]. The block has 9 cells and the Histogram of a cell has 9 bins, therefore, the block vector is 81 size long vector. To normalize the concatenated vector of the block, each element of a vector is divided by following equation 
$\sqrt{x 1^{2}+x 2^{2}+x 3^{2} \ldots \ldots+x 81^{2}}$. Where $x(1,2 \ldots n)$ are the elements of the vector. The final feature vector is the summation of the vectors of all the cells and blocks, which can be represented by $\{(l \times m) \times n\}$, where $l$ is the number of bins, $m$ is the number of cells in each block, and $n$ is the number of blocks in the image. For example, if $l$ is $9, m$ is 9 , and $n$ is 20 , then the feature vector size is 1620. Figure 3.9(c) shows the extracted histogram of a cell within a bolt image.

\subsubsection{CBD training using Adaboost}

After the features are extracted from the CBD's positive training dataset, Adaboost meta-algorithm is adopted for building the classifier based on extracted features of the bolt (Figure 2.1).

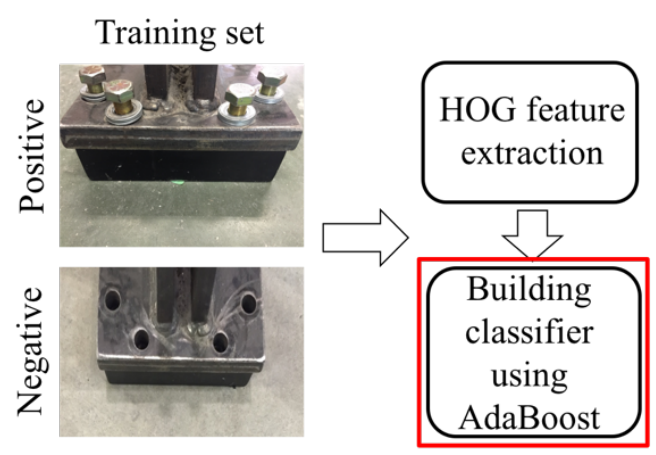

(a) CBD training

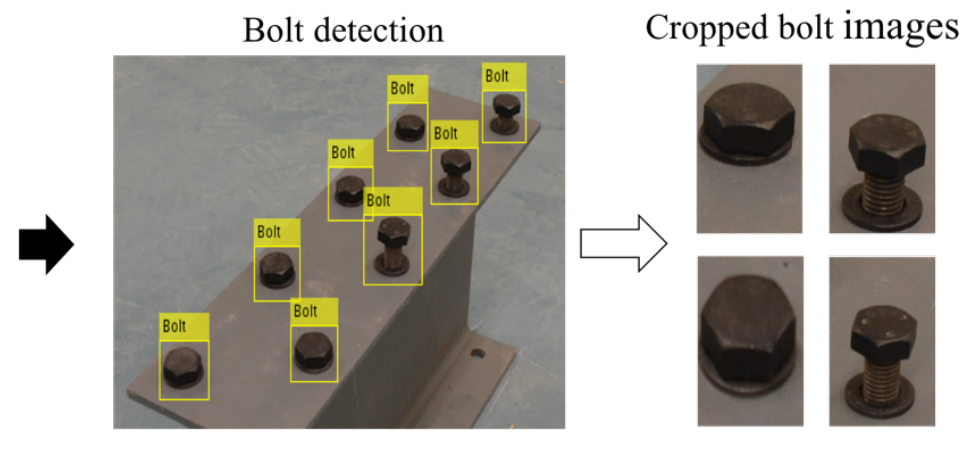

(b) CBD testing

Figure 3.10. This section refers to building classifier using Adaboost stage in training.

The Adaboost algorithm works on the principle of the decision tree [54]. It is a machine learning algorithm widely used for classification of data. The AdaBoost gives highly accurate (i.e. strong) classifiers by combining a series of weak classifiers [62-63]. A classifier with an accuracy that is slightly greater than a random guess is termed as a weak classifier [54]. The weak classifier in 
CBD are called as decision stumps [64]. Decision stumps are single node decision trees, such as they classify data into two classes. For example, classification has to performed on data of 100 humans with a height more than $1.6 \mathrm{~m}$, so for humans with a height more than $1.6 \mathrm{~m}$, class will be labelled +1 , otherwise -1 . To train the CBD classifier, the training dataset mentioned in the beginning of this section is used. The images for the training dataset were taken with a DSLR camera at varying distances and angles to include different bolt orientations and thus improve the accuracy of the CBD.

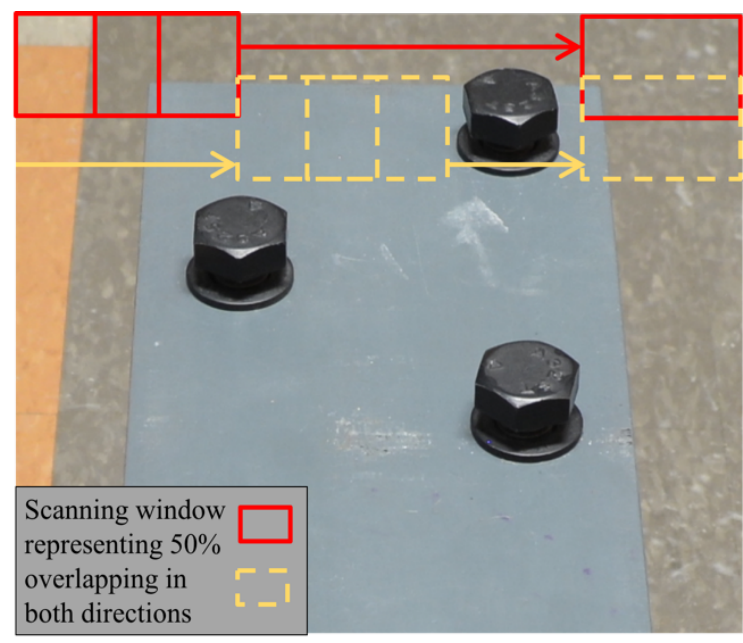

(a) Scanning window slides over the image to detect bolts

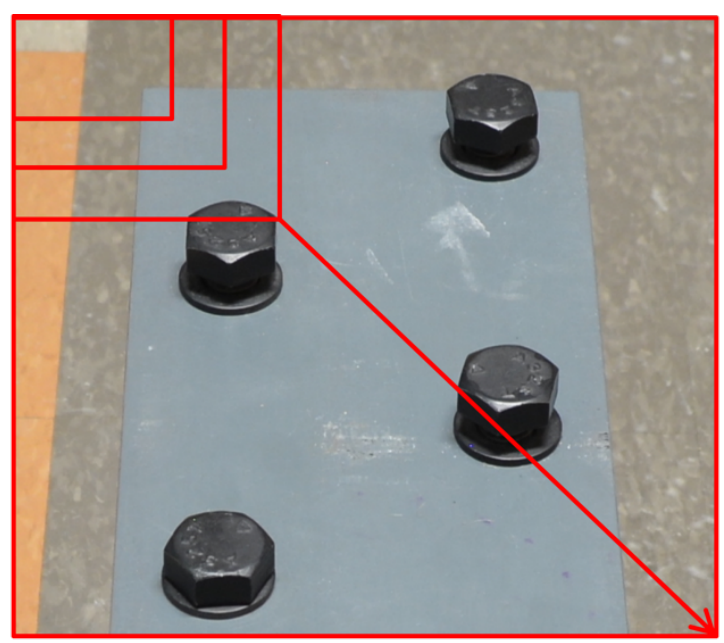

(b) Represents increase in size of scanning window

Figure 3.11 - Simplified view of scanning window over the image for detecting bolts

This algorithm uses a moving scanning window to identify positive and negative images within it, as shown in Figure 3.11(a). The sliding window technique is widely used image processing. The algorithm automatically picks the smallest size among the positive dataset for the initial window size. For example, in this study, the smallest positive image is $100 \times 100$ pixels; thus, the initial size of the scanning window is equal to $100 \times 100$ pixels. From a given training dataset $D=$ 
$\left(A_{i}, B_{i}\right)$, where $i=1,2, \ldots, N$, and $A_{i}$, and $B_{i}$ are the image and its label (positive or negative), respectively, which are prepared manually; $N$ is the total number of the training images. Figure 3.11(a) (i.e. the original image) shows the scanning window for detecting objects based on the HOG features. It uses a $50 \%$ overlapping method to avoid the incorrect classification of positive images that are at the edge of the scanning window (Figure 3.11(a)). Also, the scanning window size increases by 1.25 times after scanning the image at one scale and keeps increasing until equals the size of the image or vertical or horizontal array of the image as shown in Figure 3.11(b).

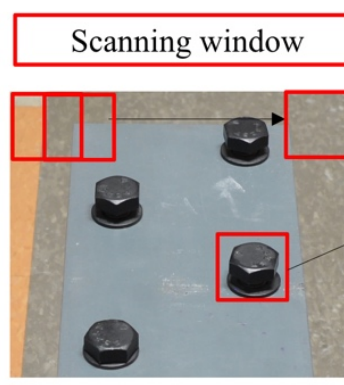

(a) Image scanning

Positive images Negative images

Weak classifier 1 Neak classifier 2 - Weak classifier $3---$

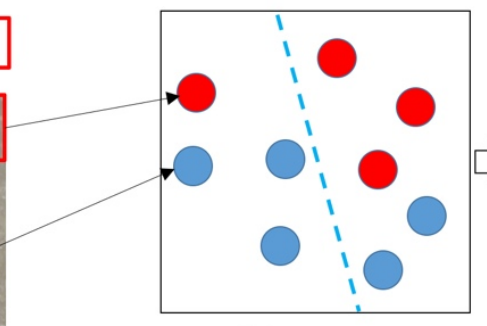

(b)

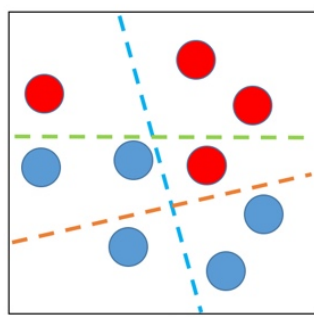

(g)

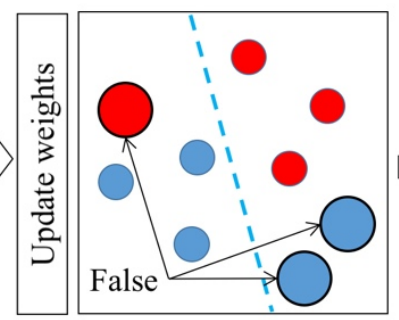

(c)

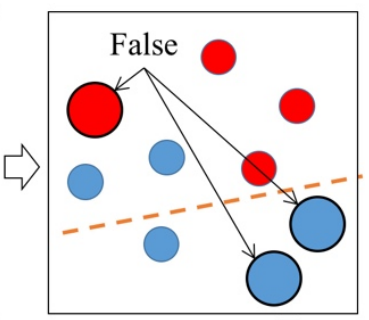

(d)

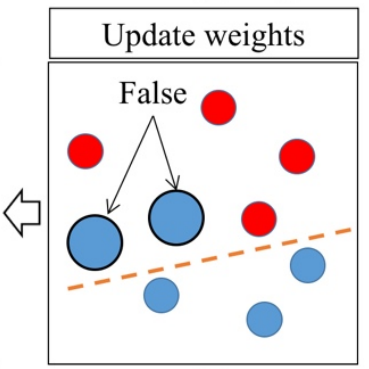

(e)

Figure 3.12 - Schematic diagram explaining working of Adaboost

Figure 3.12 explains the schematic diagram of Adaboost working. The first weak classifier performs classifications on positive and negative images from the training dataset provided and generates its decision hypothesis based on the extracted HOG features, as shown in Figure 3.12(b) [62]. The images are labelled positive $(+1)$ if the bolt is detected, otherwise, negative $(-1)$. The 
weak classifier is partially better than random guessing. Therefore, all the bolts in the positive dataset will not be detected and are labelled as false classifications or negative images are labelled as positives (Figure 3.12(c)). After evaluation by the first classifier, the weight of false classifications is increased, whereas, decreased for true classifications, as shown in Figure 5(c). In this way false classifications are prioritized over true classifications. This step refers to the adaptive boosting because initially, the weight of all the images are equal. Updating the weight based on classification makes Adaboost standout from other classifiers. Figure 5 (b-f) represents the series of weak classifiers. The final classifier is formed by combining all the weak classifiers, as shown in Figure $5(\mathrm{~g})$. The detection accuracy of the final classifier is indeed better compared to the individual weak classifiers, and only the final classifier is used for bolt detection in testing. The hypothesis given by a strong classifier made from the combination of all weak classifiers is given by Equation (3):

$$
\begin{gathered}
C(x)=\operatorname{sign}\left(\sum_{t=1}^{T} \alpha_{t} h_{t}(x)\right) \\
C(x)>0(\text { class is positive }) ; C(x)<0(\text { class is negative }),
\end{gathered}
$$

where $C$ represents the final classifier, $t$ represents the number of iterations, $h_{t}$ is the hypothesis of weak classifier, and $\alpha_{t}$ is the coefficient calculated from the error $\left(\varepsilon_{t}\right)$ of hypothesis $\left(h_{t}\right)$ of each weak classifier given by Equation (4), where error $\left(\varepsilon_{t}\right)$ is the number of misclassifications divided by the dataset for each classifier.

$$
\alpha_{t}=1 / 2\left(\frac{1-\varepsilon_{t}}{\varepsilon_{t}}\right)
$$


Prior to the classification of the first weak classifier, the initial weights of all the images are the same and updated after the classification by each weak classifier using equation (5):

$$
w_{t+1}\left(A_{i}\right)=w_{t}\left(A_{i}\right) e^{ \pm \alpha_{t}}
$$

where $w_{t}$ is the weight at $t^{\text {th }}$ iteration, and $w_{t+1}$ is the weight for the next iteration. If the label of an image $B_{i}$ is true for a hypothesis, the weight is decreased by $e^{-\alpha_{t}}$, otherwise the weight is increased by $e^{+\alpha_{t}}$. The training is completed if the algorithm reaches the specified number of stages, and the CBD is trained through 20 stages. However, the algorithm halts the training if the classifier's error is more than $50 \%$ or a sufficient number of negative images are not available. A possible reason for the former is that the training data may not have invariant features. As a result, the algorithm cannot generate an effective hypothesis. Therefore, a well-annotated dataset is essential. For the later, providing more negative images or reducing the number of stages are possible solutions, but this may also cause a deterioration of the accuracy. The algorithm iteratively generates classifiers at each stage, where the number of negative images is defined by the negative sample factor. If the factor is three, then three negative images corresponding to each positive image are used at each stage. The factor used for training CBD is two.

The algorithm moves to the next stage if one of the following conditions are satisfied. First, the number of weak classifiers is equal to 100 . Second, the false positive rate reaches a defined value between 0 to 1 . If we set this value to zero then we are expecting very high accuracy from initial weak classifiers, which may lead to false detections. For this study, a false positive rate of 0.4 is used. Lastly, the detection error decreases to zero. The aforementioned parameters can only be 
configured by trial and error because they depend on the features of objects. During training, the scanning window labels the regions as positive or negative based on the detection. All the negative label regions are discarded whereas the positive regions are passed to the subsequent stages. Each successive stage is more complex than the previous because the classifiers use more features than the previous stages to discard more false positives and detect all the true positive regions. Hence, initial stage will have more false positives with all true positives and subsequent stages major contribution is to eliminate the false positives and detecting all true positives. In other words, Adaboost at every stage discards more non object images than a former stage. The positive images, which pass through all the stages, are labeled as objects, and the rest are rejected as shown in Figure 3.13 [65-66].

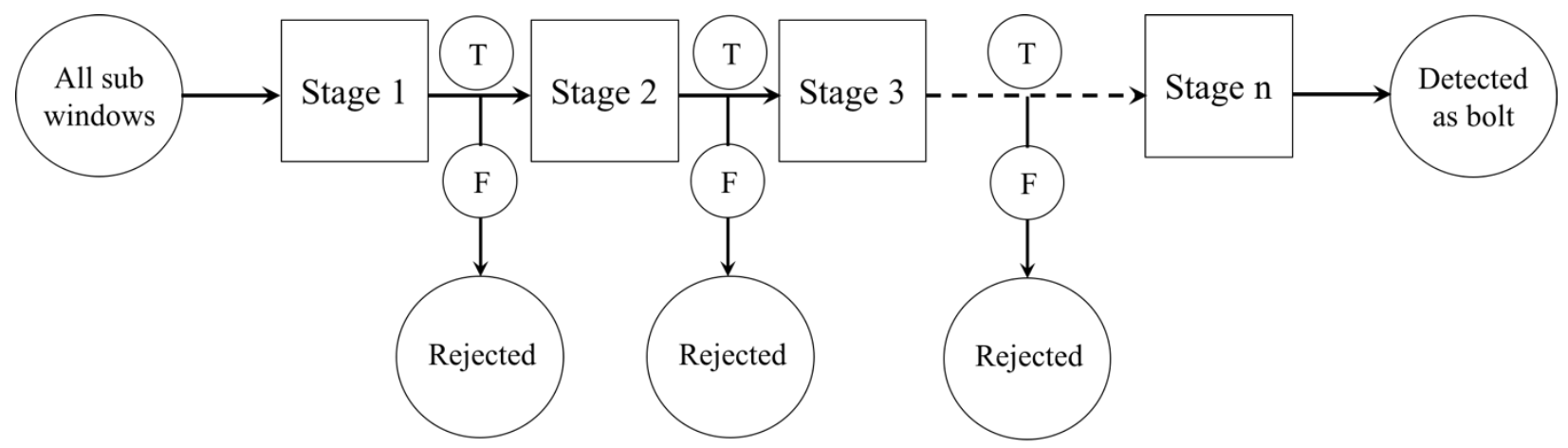

$\mathrm{T}$ - true positive and false positive F - true negative and false negative

Figure 3.13. Explains how sub windows proceed through different stages based on the bolt detection. 


\subsection{Support vector machine (SVM) classifier}

As stated in the beginning of this chapter, the proposed methodology consists of two methods: CBD as explained above, followed by SVM briefed in this part of the chapter. The CBD's role is to detect bolts and crop the isolated bolt images. The SVM classifier generates a hyperplane to differentiate two classes from the input data based on the extracted features of the object. In bolt classification, the two classes refer to loosened and tight bolts. As shown in Figure 3.14, SVM classifies data into two classes +1 and -1 [67], where ' $m$ ' is margin, shortest distance between hyperplane and points of each class, which are also called support vectors. The bigger the margin, the better the SVM performance [61]. The SVM decision boundary is given by:

$$
w^{T} x_{n}+b
$$

where $w^{T}$ is weight vector orthogonal to hyperplane, $x_{n}$ input data and $\mathrm{b}$ is bias of the dataset.

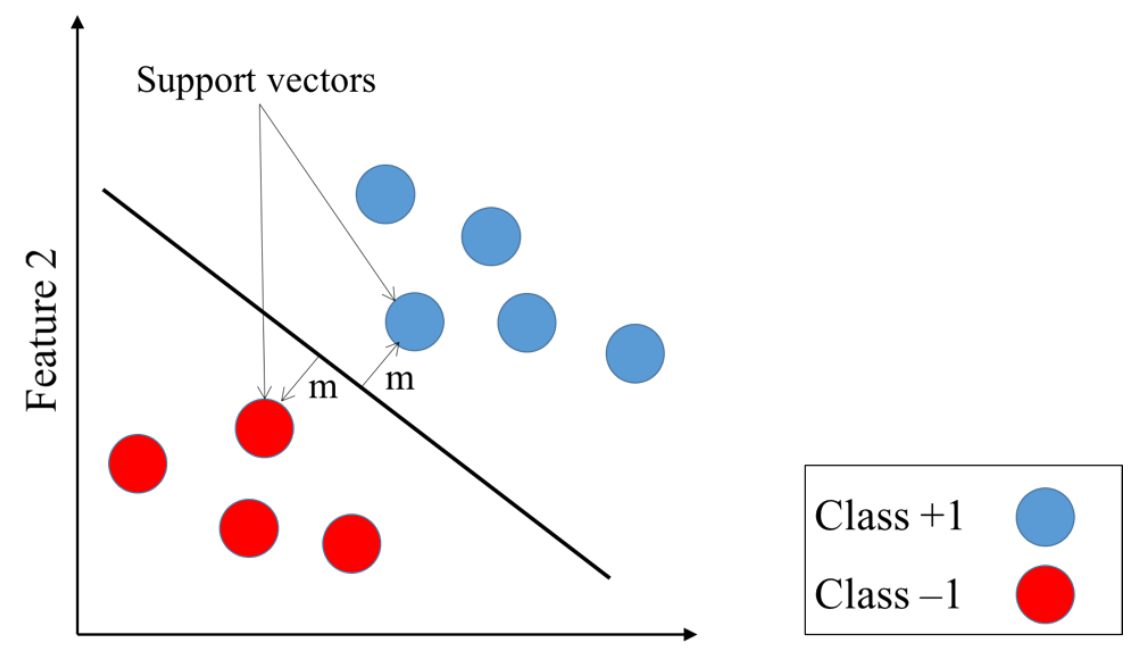

Feature 1

Figure 3.14. Illustration of SVM classifier with hyperplane differentiating data classes.

$$
t_{n}=+1 \text { for } w^{T} x_{n}+b \geq 1 ; t_{n}=-1 \text { for } w^{T} x_{n}+b \leq 1
$$


Where, $t_{n}$ is the class of the data points provided for training the SVM. For better accuracy, SVM seek to maximize the margin. From deriving equation (7), term to maximize the margin is generated.

$$
m=\frac{1}{\|w\|}
$$

In other words, minimizing $w$ and bias $b$ will generate the optimal hyperplane. Which is achieved through Langrangian equation.

$$
L=\frac{1}{2} w^{T} w-\sum_{n=1}^{N} \alpha_{n}\left(t_{n}\left(w^{T} x_{n}+b\right)-1\right)
$$

Where $\alpha$ is Lagrange multiplier. Partial derivatives of this equation w.r.t $w$ and $b$ and substituting back in equation (9) gives:

$$
\begin{gathered}
t_{\text {new }}=\operatorname{sign}\left(\sum_{n=1}^{N} \alpha_{n} t_{n} x_{n}^{T} x_{\text {new }}+b\right) \\
b=t_{n}-\sum_{m=1}^{N} \alpha_{m} t_{m} x_{m}^{T} x_{n}
\end{gathered}
$$

In equation (11) $x_{n}$ is the closet data point or the support vector. The trained SVM uses equation (10-11) for classifying any data point fed.

\subsubsection{SVM training dataset}

After training the CBD, next step is to train the SVM for classification of loosened and tight bolts. SVM is trained on another dataset of loose and tight bolt images as shown in Figure 3.15. SVM's training dataset consist of 17 tight and 14 loosened bolts, where the training dataset is completely independent of the test dataset. The images for the training dataset consists of varied loosened length of the bolts $(5 \mathrm{~mm}-25 \mathrm{~mm})$, also varying vertical and horizontal angle for the loose and tight sets. 


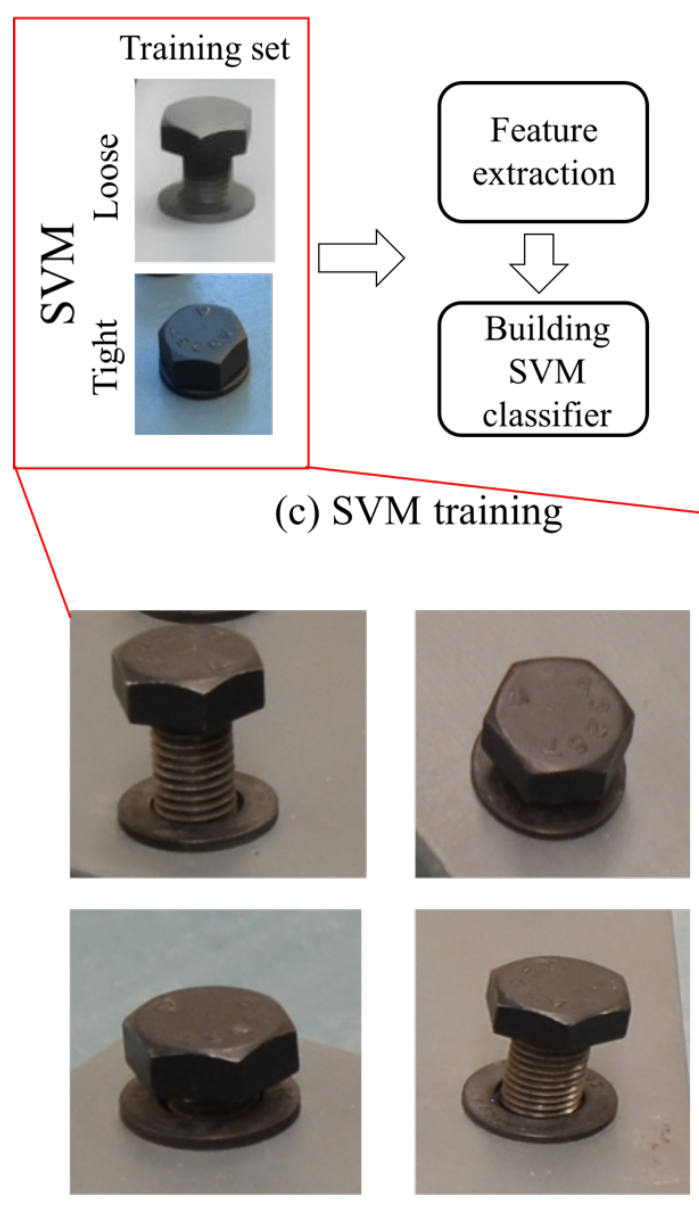

Loosened bolt images

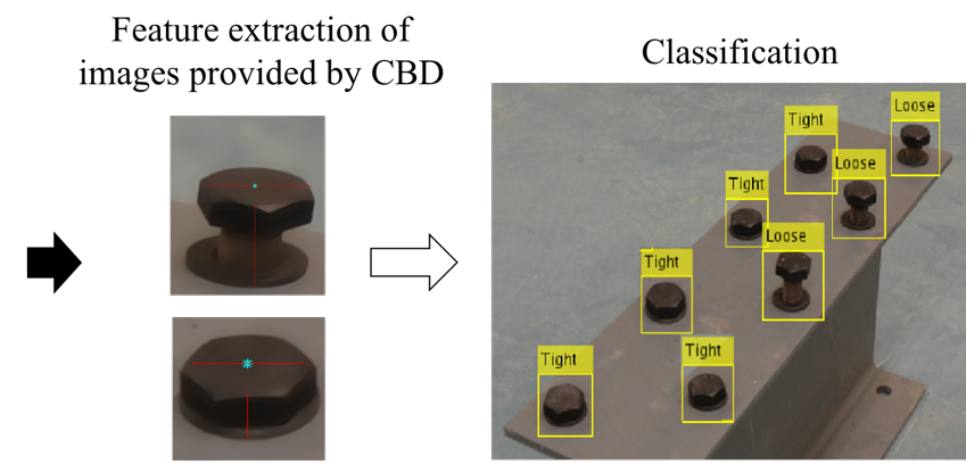

(d) SVM testing

Figure 3.15. Training dataset for SVM training.

\subsubsection{Feature extraction}

The SVM classifier adopts a previously introduced method [53] with a few modifications, which are detailed in this section. The SVM classifier is trained on bolt features. The following features are considered: (1) the width of the bolt head $(l)$; (2) the vertical length or half the minor-axis of the ellipse on the bolt head $\left(h^{\prime}\right)$; and (3) the exposed thread length of the bolt $(h)$, as shown in Figure 3.16(a). Also, Figure 3.16 shows the flow of the feature extraction process of the proposed method. The width $l$ and $h^{\prime}$ are extracted from detected ellipse parameters, whereas the binary 
detector is used for measuring $h$ as shown in Figure 3.16(b, c, d). The shape of the bolt head and the washer resemble an ellipse and are detected using Hough's transform [68]. The five major components of an ellipse are two coordinates of centroid, major axis, minor axis, and orientation. The major axis of the best fitted ellipse is directly used as width of the bolt head, whereas half the minor axis of the best fitted ellipse is used as $h^{\prime}$. The binary detector moves downward pixel-bypixel, starting from the minor axis of bolt head and stops at the encounter of white pixel in the background measuring $h$ (Figure 3.16(c)). The feature extraction method proposed by Cha et. al. [53] struggles to find ellipses fitting the bolt heads because the Hough transform detects some of the pixels in the image background as part of the ellipses [68-69]. To overcome this issue, contrast adjustment was adopted as shown in Figure 3.17 [70]. For detecting the ellipses fitting the bolt head, the images are denoised and the contrast is enhanced between foreground and the background, resulting in improved detectability of ellipses.

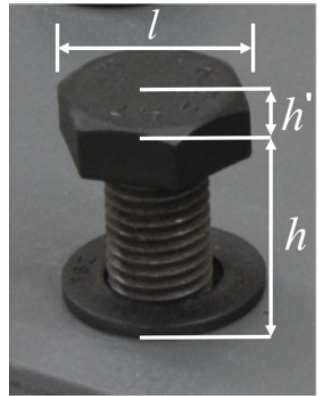

(a) Bolt features

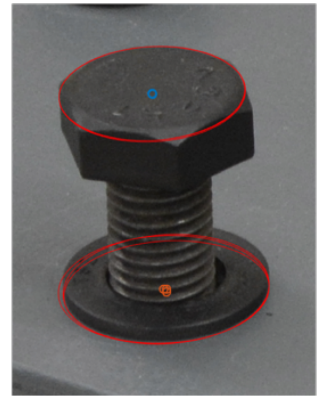

(b) Ellipse detection

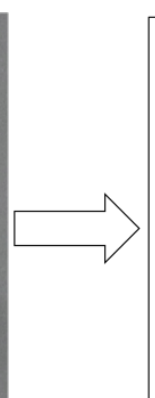

(c) Binary detector

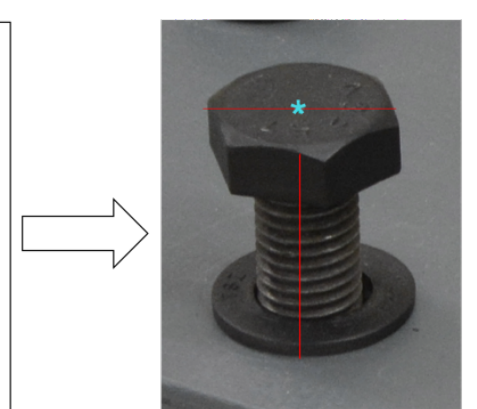

(d) Calculated features

Figure 3.16. (a) Represent bolt features used for classification (b) represent the ellipse detection over the bolt head (c) binary detector for calculating the vertical height of the bolt $(h)$ and $(d)$ shows the calculated bolt features from the ellipse parameters and binary detector. 
Another issue related to the previous method was, hollows in the binarized images of the bolts, as shown in Figure 3.17(c). These hollows are caused by different light intensities or reflections on the bolt surface and hinder the accurate calculation of $h$, which leads to false classifications. Since the binary detector stops at the encounter of white pixel, the hollows on either bolt head, shank, or washer can lead to false calculation of the vertical height feature $h$, as shown in the Figure 3.17 the red dashed box. This issue has now been eliminated by adopting a masking technique [71], and the modified algorithm can segment the bolts from background effectively.

(a) Gray scale image

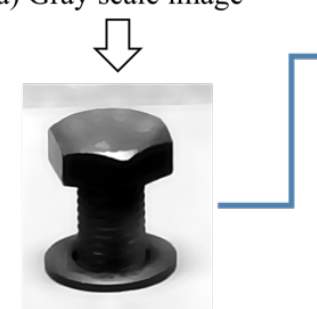

(b) Contrast adjusted gray scale image

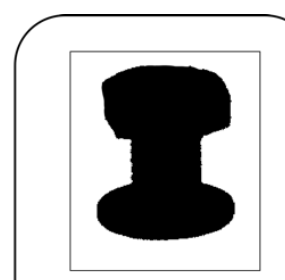

(d) Binary image using masking

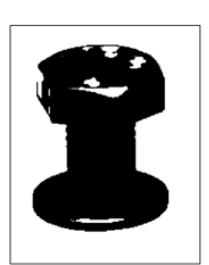

(c) Binary image
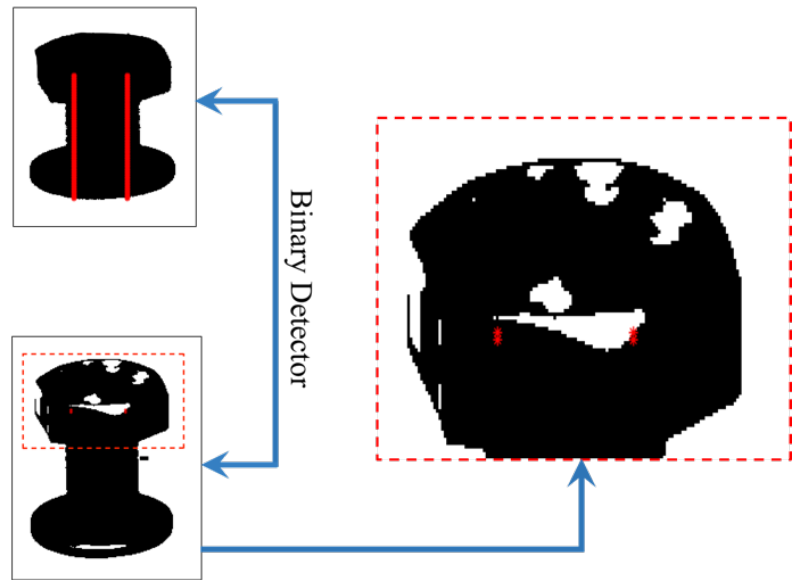

Figure 3.17. Shows the contrast enhancement and masking of the binary image for accurate feature calculation.

\subsubsection{Building a SVM classifier}

Even if the physical conditions of the bolts are the same, the bolts have varying features in image spaces due to different image taking conditions (distance and angles). Therefore, for bolt classification, ratios of $l / h, l / h^{\prime}$, and $h^{\prime} / h$ are considered instead of $l, h^{\prime}$, and $h$. In Figure 3.18, the 
loose bolt images are taken at more distance from the camera lens than the tight bolt images. Since both the images have the same dimensions, the binary detector calculates the feature through pixelby-pixel movement. Therefore, the vertical height feature $h$ on the loose bolt images is quite similar to the tight bolts images. If these images are classified based on these features directly, it will generate more false classifications and the results will be misleading. Realistically, not all the bolts in the image are at the same distance and vertical angle. To overcome the issue of vertical angle and distance variations, instead of using the bolt features, direct ratios of the extracted features were used for classification. In Figure 3.18, feature $l$ and $h^{\prime}$ are different. Hence, using the ratios $l / h, l / h^{\prime}$, and $h^{\prime} / h$ as the feature for classification generates more accurate results.

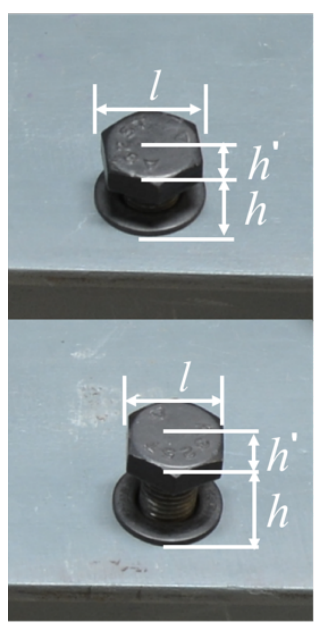

Loose bolts

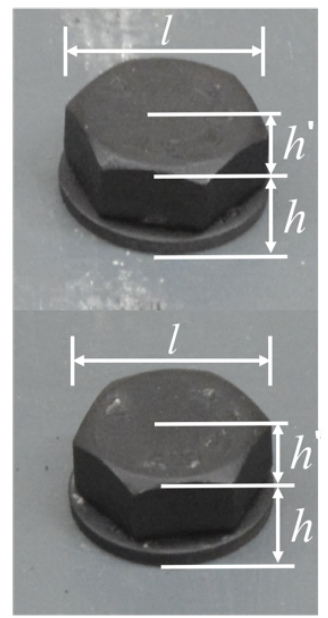

Tight bolts

Figure 3.18. Bolt feature comparison between loose bolts at farther distance and tight bolts at lesser distance.

All the training images were manually cropped and carefully chosen in order to set a decision boundary for the SVM that effectively classifies the test images. Figure 3.19 shows the trained 
SVM, where the gray hyperplane separates the tight and loosened bolt features; this hyperplane is used for bolt classifications in the test dataset, which is detailed in Section 4.

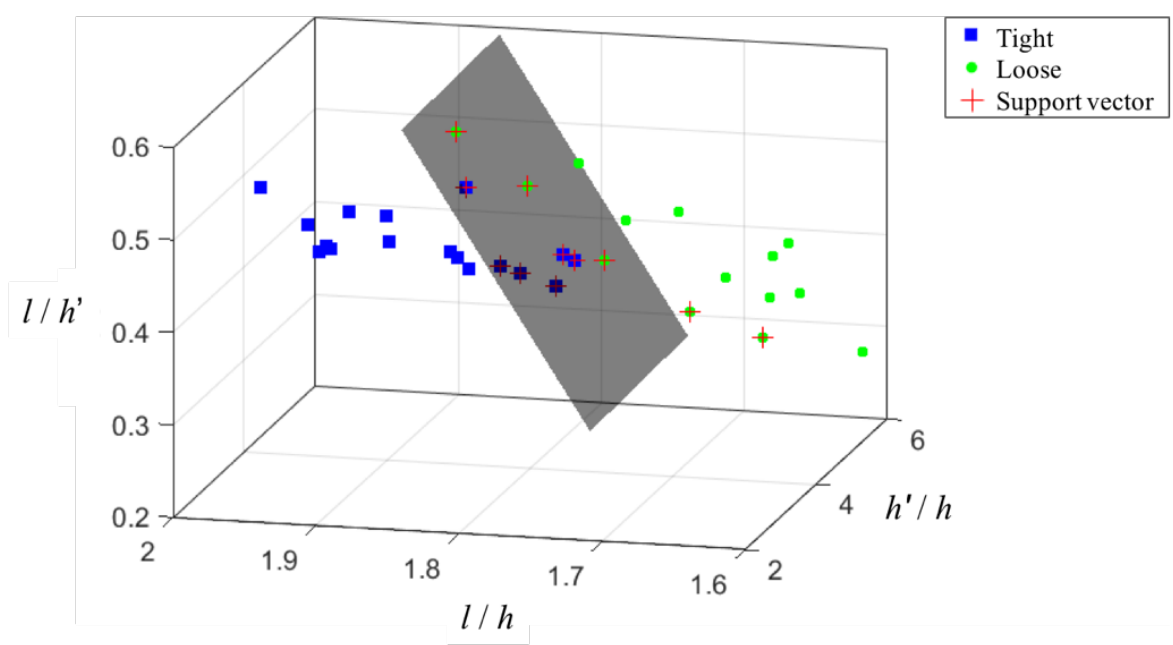

Figure 3.19. Trained SVM. 


\section{Chapter 4 - Validation}

\subsection{Experimental setup}

The proposed method is motivated from the Cha et. al. [53] study by removing the limitations. The methodology was tested on steel I-section with eight bolts of varying looseness or tightness, and varying vertical and horizontal angle. Figure 4.1(a) shows the experimental setup details with specimen and camera, where;

- $\quad \mathrm{L}$ is the horizontal distance of the camera from the specimen centroid

- $\mathrm{H}$ is the height of the camera lens from ground level

- $\mathrm{A}_{\mathrm{h}}$ is the horizontal angle of the camera from the centroid of specimen

- $\mathrm{A}_{\mathrm{V}}$ is the vertical angle of the bolt

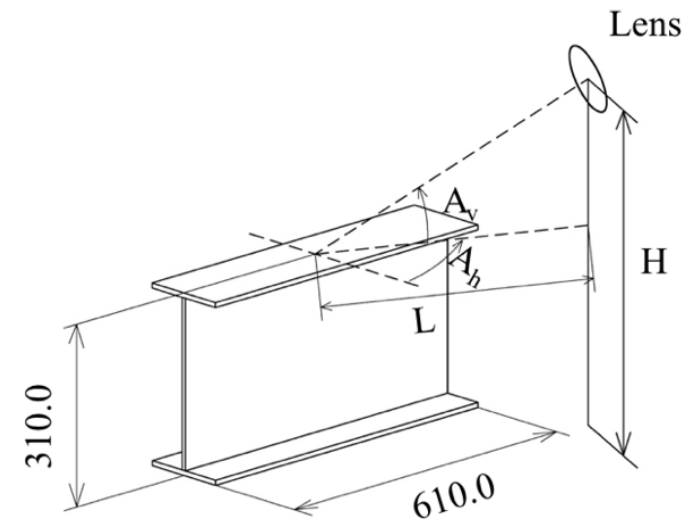

(Unit: mm)

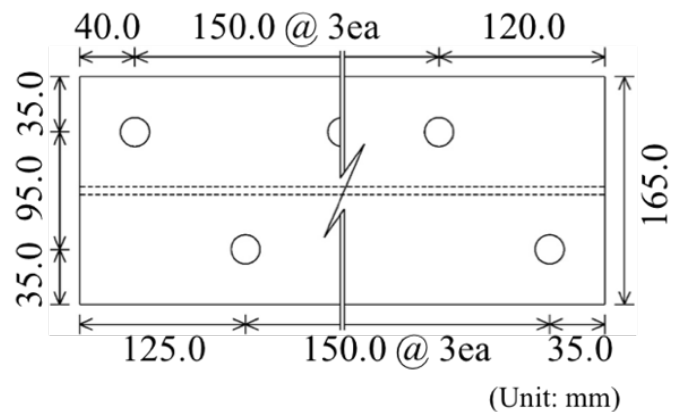

(b)

Figure 4.1. (a) Experimental setup (b) bolt details on the specimen. 
The vertical angle $A_{v}$ is calculated by subtracting the height of the specimen from H. Figure 4.1(b) demonstrates the staggered bolt layout with $150 \mathrm{~mm}$ pitch, $95 \mathrm{~mm}$ gauge, and edge distance as shown. M20 hexagonal structural bolts were used for the experiment. The test images were taken in Agriculture engineering building's hallway.

\subsection{Experimental study}

The length of the I-section specimen used for this study was $610 \mathrm{~mm}$. The bolt images were taken from four horizontal angles corresponding to every vertical angle as shown in Table 4.1. Total 198 bolt images were taken at $4000 \times 6000$ resolution using a Nikon D5200 DSLR camera.

Table 4.1. Images were taken at combination of vertical and horizontal angle

\begin{tabular}{ccc}
\hline $\mathrm{A}_{\mathrm{v}}=\operatorname{Tan}^{-1}\left(\mathrm{H}^{\prime} / \mathrm{L}\right) ;$ & & \\
$\mathrm{L}=1000 \mathrm{~mm} ;$ & $\mathrm{A}_{\mathrm{h}}$ & \\
$\mathrm{H}^{\prime}=(\mathrm{H}-310) \mathrm{mm}$ & $0^{\circ}, 30^{\circ}, 60^{\circ}, 90^{\circ}$ & @ each angle \\
\hline $\mathrm{H}^{\prime}=270 \mathrm{~mm} ; 15^{\circ}$ & $0^{\circ}, 30^{\circ}, 60^{\circ}, 90^{\circ}$ & combination 2 images \\
$\mathrm{H}^{\prime}=365 \mathrm{~mm} ; 20^{\circ}$ & $0^{\circ}, 30^{\circ}, 60^{\circ}, 90^{\circ}$ & are taken with random \\
$\mathrm{H}^{\prime}=490 \mathrm{~mm} ; 26^{\circ}$ & $0^{\circ}, 30^{\circ}, 60^{\circ}, 90^{\circ}$ & loose and tight bolts \\
$\mathrm{H}^{\prime}=580 \mathrm{~mm} ; 30^{\circ}$ & $0^{\circ}, 30^{\circ}, 60^{\circ}, 90^{\circ}$ & locations \\
$\mathrm{H}^{\prime}=730 \mathrm{~mm} ; 36^{\circ}$ & $0^{\circ}, 30^{\circ}, 60^{\circ}, 90^{\circ}$ & \\
$\mathrm{H}^{\prime}=900 \mathrm{~mm} ; 42^{\circ}$ & & \\
\hline
\end{tabular}


The vertical angle was incorporated to study the effective range of this method. SVM can classify loosened and tight bolts at low vertical angles very precisely; at sharp vertical angles, the SVM cannot classify bolts correctly, arguably because at sharp vertical angles the feature $h$ becomes too small. In Figure 4.2, feature $h$ drastically reduces at $53^{\circ}$ vertical angle compared to $20^{\circ}$. As a result, the SVM might not classify small loosened length correctly. On the other hand, horizontal angles were used for accommodating the bolt layout geometry. In Figure 4.3, same bolt layout is captured at four horizontal angles but the bolts overlap at $30^{\circ}$ and $90^{\circ}$. Thus, a single horizontal angle cannot accommodate all bolt layout geometries. For example, Figure 4.3 shows a staggered bolt layout where the bolts are well distinguished at $0^{\circ}$ and $60^{\circ}$ but in case of parallel bolt layout, a $30^{\circ}$ horizontal angle will provide better results than $0^{\circ}$. Also, this issue is limited to low vertical angles; at higher vertical angles (26 and above), bolt overlapping reduces to negligible.

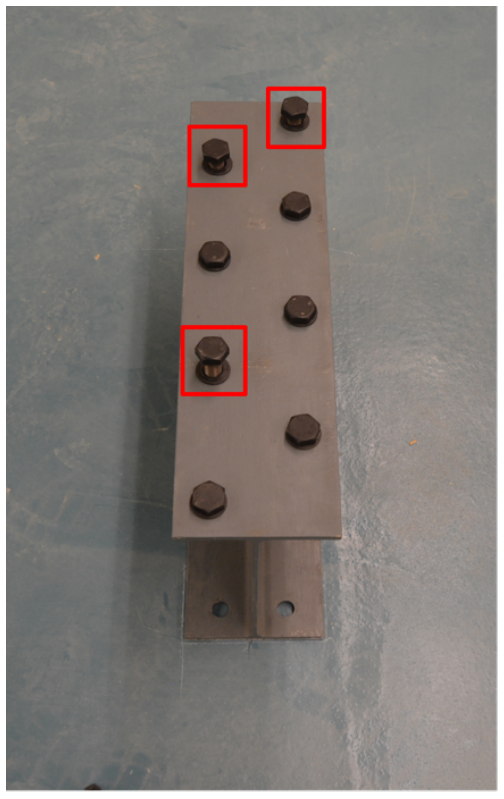

(a) $53^{\circ}$ Vertical angle

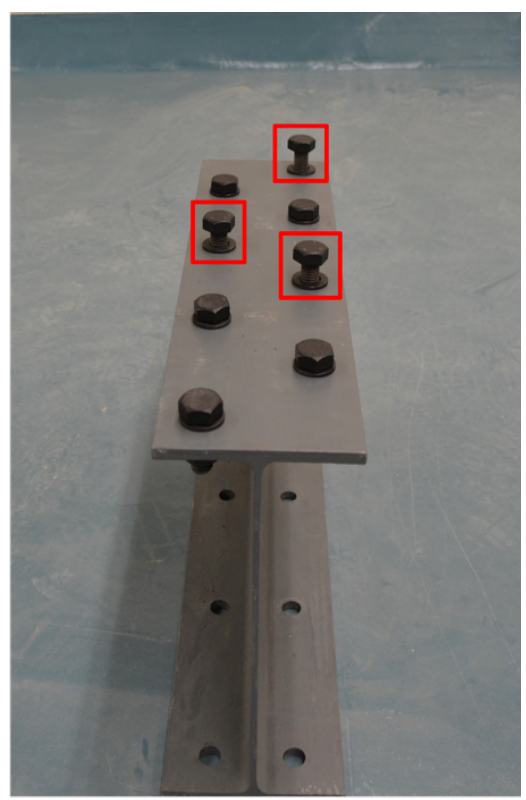

(b) $20^{\circ}$ Vertical angle

Figure 4.2. Vertical angle comparison. 


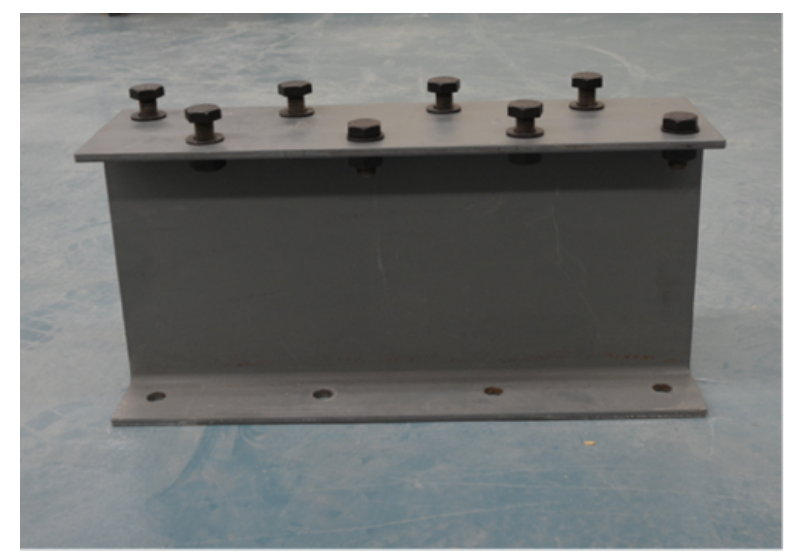

(a) $0^{\circ}$ horizontal angle

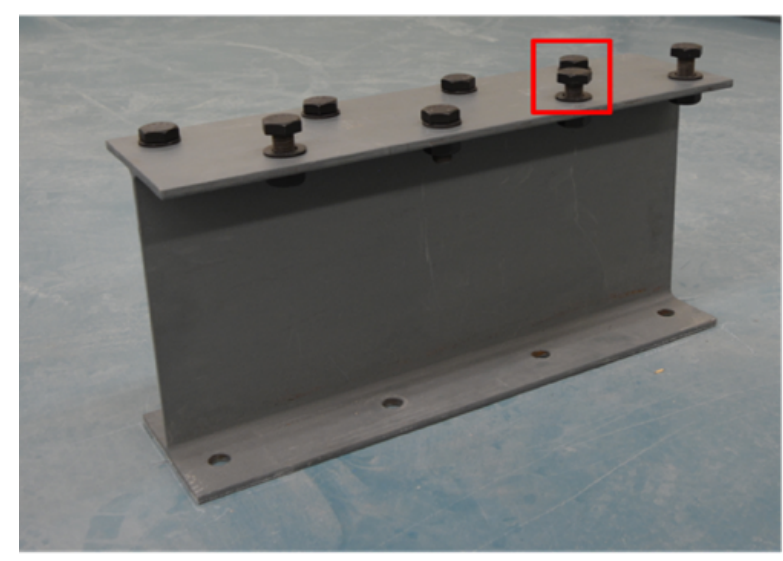

(b) $30^{\circ}$ horizontal angle

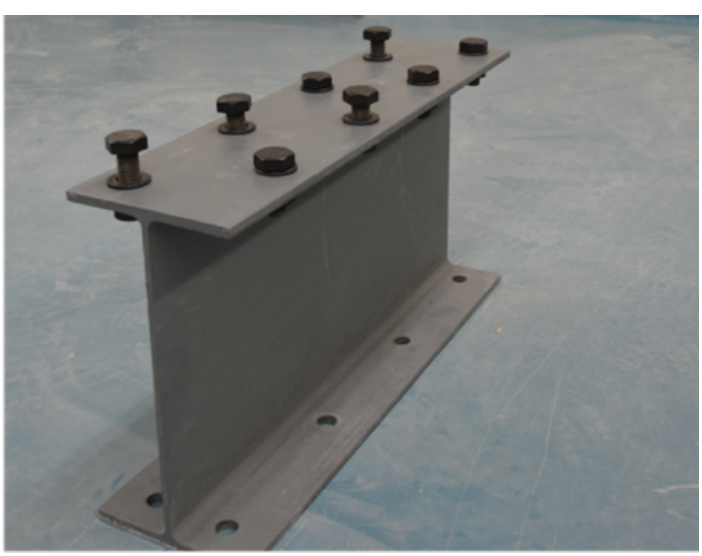

(c) $60^{\circ}$ horizontal angle

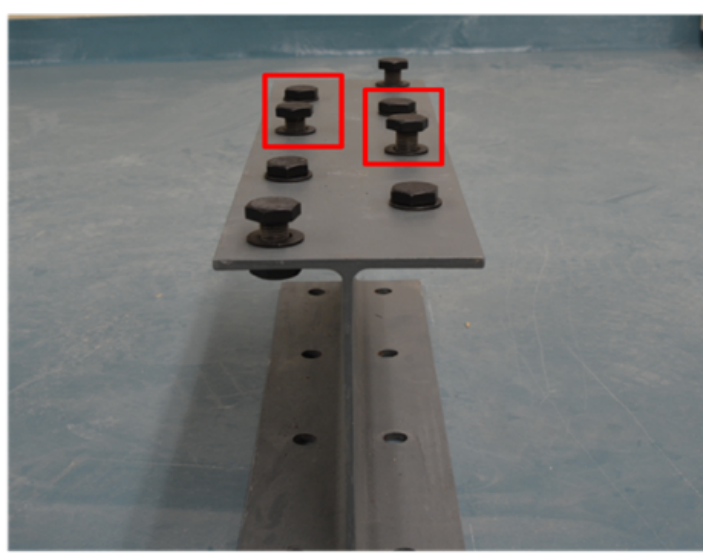

(d) $90^{\circ}$ horizontal angle

Figure 4.3. Effect on horizontal angle on bolt layout geometry.

The bolt layout and vertical angle presented in Figure 4.1 show the variation of $3^{\circ}$ to $8^{\circ}$ vertical angle variation of each bolt that occurs within the same image. Therefore, the vertical angle of each bolt is calculated to conduct the precise experimental study, as shown in Table 4.2. A total of 1584 loosened and tight bolts were tested for detection and classification. 
Table 4.2. The number of bolts according to the range of vertical angles and loosened length.

\begin{tabular}{cccccccc}
\hline $\begin{array}{c}\text { Vertical } \\
\text { angle }\left(^{\circ}\right)\end{array}$ & Tight & $5 \mathrm{~mm}$ & $10 \mathrm{~mm}$ & $15 \mathrm{~mm}$ & $20 \mathrm{~mm}$ & $25 \mathrm{~mm}$ & Sum \\
\hline $10-14$ & 16 & 18 & 17 & 29 & 34 & 23 & 137 \\
$15-19$ & 29 & 29 & 26 & 50 & 36 & 27 & 197 \\
$20-24$ & 19 & 26 & 29 & 47 & 33 & 30 & 184 \\
$25-29$ & 29 & 34 & 29 & 49 & 53 & 43 & 237 \\
$30-34$ & 23 & 29 & 33 & 37 & 36 & 39 & 197 \\
$35-39$ & 32 & 33 & 33 & 44 & 29 & 36 & 207 \\
$40-44$ & 40 & 47 & 44 & 36 & 37 & 38 & 242 \\
$45-51$ & 31 & 33 & 32 & 28 & 27 & 32 & 183 \\
Sum & 219 & 249 & 243 & 320 & 285 & 268 & \\
\hline
\end{tabular}

\subsection{Results}

A total of 198 images at a resolution of $6000 \times 4000$ with eight bolts in each image were tested and the results evaluated. First, the cascade classifier detects and crops the localized images of the bolts from the test images. Second, the SVM classifies the loosened and tight bolts from of the total cropped images generated by the cascade detector. Hence, the accuracy of both methods is calculated as well as the overall performance. Moreover, the accuracy of the SVM is calculated from the total number of bolts correctly cropped by CBD as the SVM cannot classify images partially cropped by the CBD. 


\subsubsection{Accuracy in bolt detection}

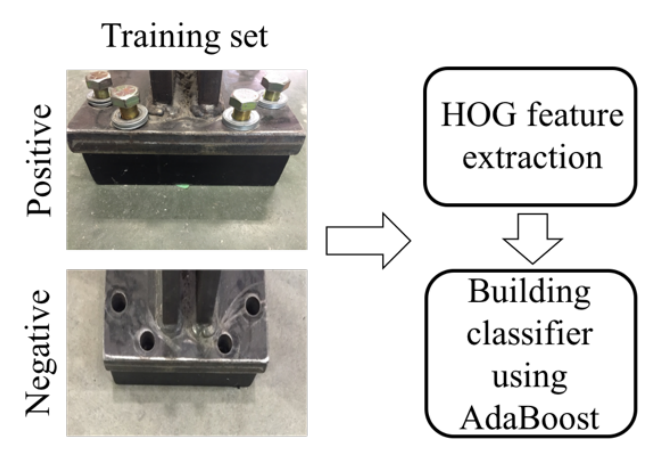

(a) CBD training

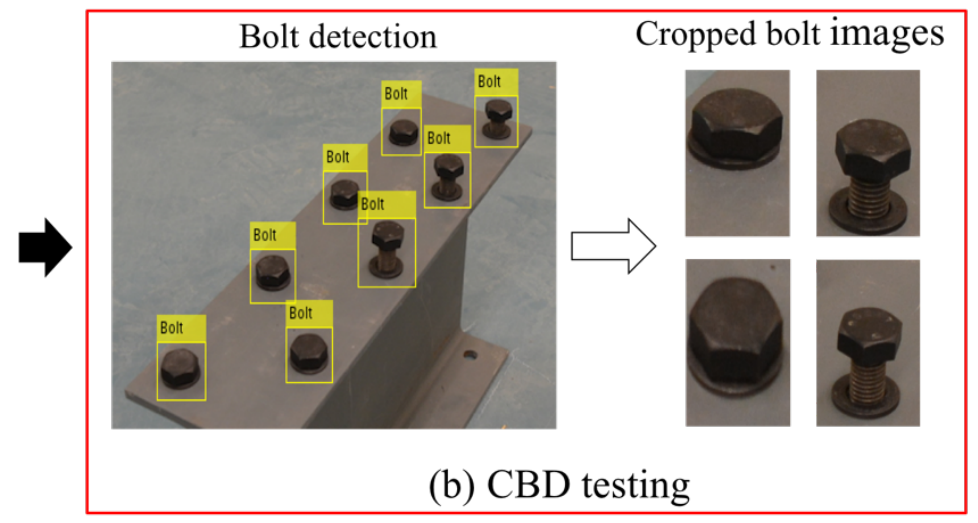

(b) CBD testing

Figure 4.4. This section is focused on CBD accuracy.

CBD false detections consist of undetected or partially detected bolts. The background detections were neglected because we want to calculate bolt features for classification. As long as the dataset for the feature calculation has all the bolts localized and cropped, the evaluation of the loosened and tight bolts can be done. In Figure 4.5, the bolts highlighted with a solid red line are partially detected and are considered as false detections. Whereas, the detections highlighted in dashed red and solid orange are neglected because they do not affect the accuracy of the CBD. Table 4.3 shows the accuracy of the CBD for all 1584 bolts. The average accuracy of the CBD is approximately 97\%. CBD accuracy is lower at low vertical angles because of bolt overlapping. As the vertical angle increases, the detection accuracy also increases because bolt overlapping decreases with an increase in vertical angle. Moreover, at low vertical angles, tight bolts fall behind $25 \mathrm{~mm}$ loose bolts, which also leads to no detection of the bolt. 


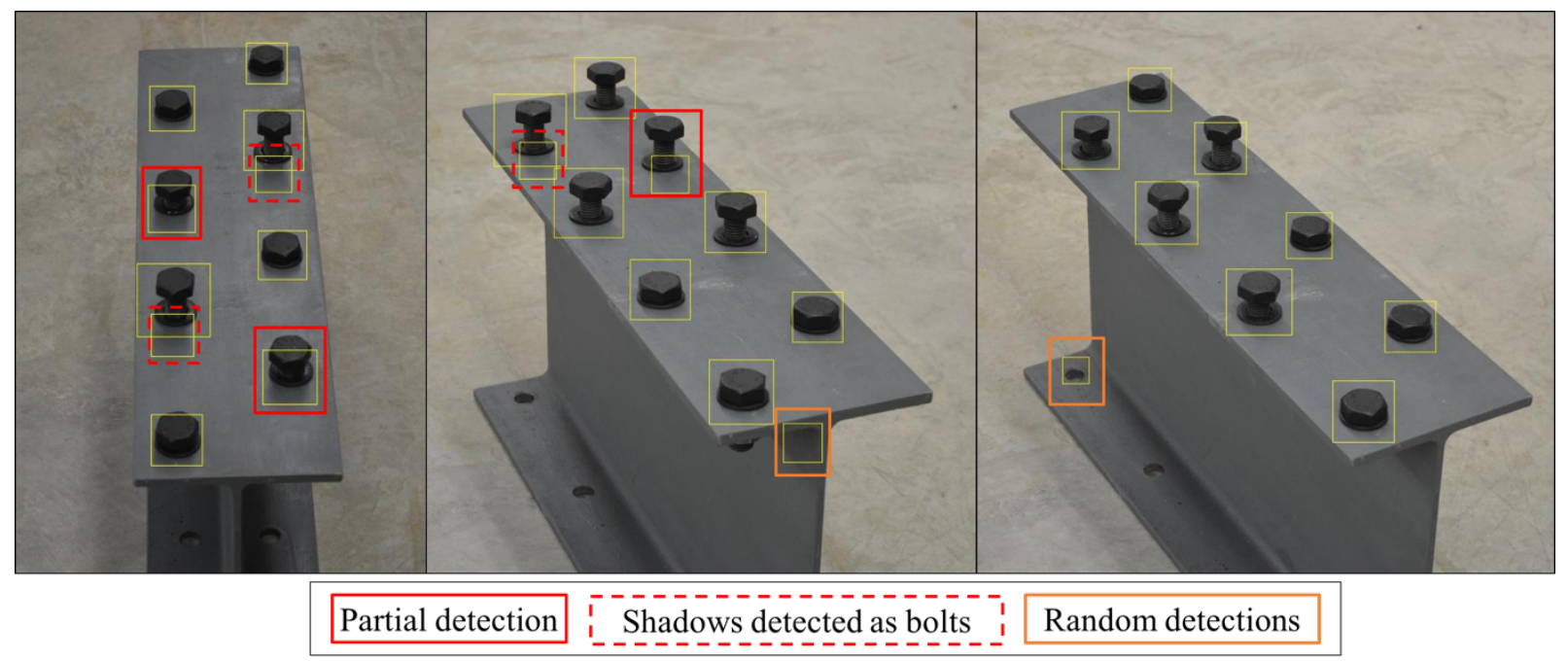

Figure 4.5. Partial detections, shadows effect and false detections by CBD.

Table 4.3. Accuracy of the CBD for loosened and tight dataset.

\begin{tabular}{|c|c|c|c|c|c|c|c|}
\hline \multirow[t]{2}{*}{ Vertical angle $\left(^{\circ}\right)$} & \multicolumn{6}{|c|}{ Loosened length } & \multirow[t]{2}{*}{ Average } \\
\hline & Tight & $5 \mathrm{~mm}$ & $10 \mathrm{~mm}$ & $15 \mathrm{~mm}$ & $20 \mathrm{~mm}$ & $25 \mathrm{~mm}$ & \\
\hline $10-14$ & 0.56 & 1.00 & 0.94 & 0.97 & 0.91 & 0.70 & 0.86 \\
\hline $15-19$ & 0.93 & 0.90 & 0.88 & 0.96 & 0.92 & 0.74 & 0.90 \\
\hline $20-24$ & 0.95 & 1.00 & 1.00 & 1.00 & 1.00 & 0.97 & 0.99 \\
\hline $25-29$ & 0.97 & 1.00 & 1.00 & 1.00 & 1.00 & 1.00 & 0.99 \\
\hline $30-34$ & 1.00 & 1.00 & 1.00 & 1.00 & 1.00 & 1.00 & 1 \\
\hline $35-39$ & 1.00 & 1.00 & 1.00 & 1.00 & 1.00 & 1.00 & 1 \\
\hline $40-44$ & 1.00 & 1.00 & 1.00 & 1.00 & 1.00 & 0.97 & 0.99 \\
\hline $45-51$ & 0.87 & 1.00 & 1.00 & 1.00 & 1.00 & 1.00 & 0.98 \\
\hline Average & 0.93 & 0.99 & 0.98 & 0.99 & 0.98 & 0.94 & \\
\hline
\end{tabular}




\subsubsection{Accuracy of the SVM classification}

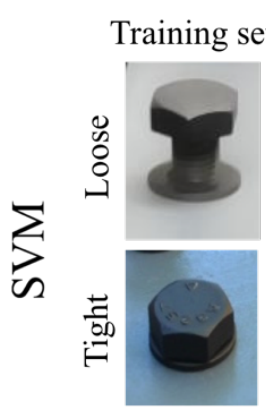

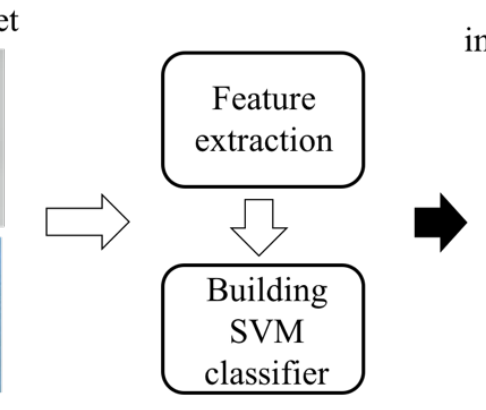

(c) SVM training

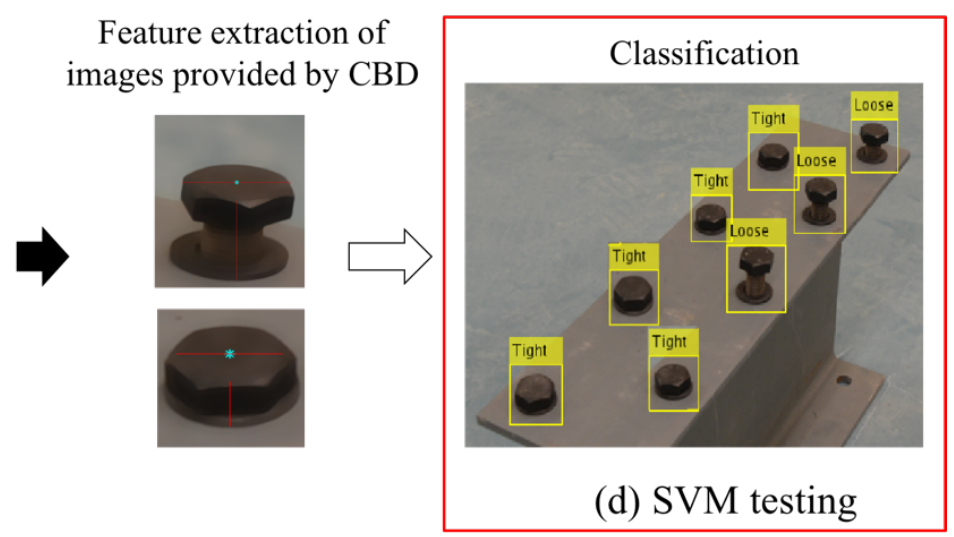

Figure 4.6. This section is focused on SVM classification accuracy of images provided by CBD.

The SVM accuracy is based on 1536 bolt images cropped by the CBD. The classification accuracy is shown in Table 4.4; the average accuracy of the SVM is $93 \%$. The classification accuracy is minimum at a $45^{\circ}-51^{\circ}$ and $40^{\circ}-44^{\circ}$ vertical angles, as illustrated in Table 3 , for tight and $5 \mathrm{~mm}$ loosened bolts. Arguably, this is because the loosened length of the bolts is sensitive to the vertical angle changes. At a sharp vertical angle, the vertical height feature becomes very sensitive. Hence, $5 \mathrm{~mm}$ loosened lengths are classified as tight, and tight are sometimes classified as loose, because of the very small margin between $5 \mathrm{~mm}$ and tight bolts. On the contrary, at a low vertical angle, the exposed shank can be observed reasonably well and therefore classification is more accurate. Additionally, a few false classifications are due to the wrong ellipse detection on the bolt head, which is a result of edge detection of the bolt head. 
Table 4.4. Accuracy of the SVM classification for the loosened and tight dataset.

\begin{tabular}{cccccccc}
\hline \multirow{2}{*}{ Vertical angle $\left(^{\circ}\right)$} & & \multicolumn{7}{c}{ Loosened length } & & Average \\
& Tight & $5 \mathrm{~mm}$ & $10 \mathrm{~mm}$ & $15 \mathrm{~mm}$ & $20 \mathrm{~mm}$ & $25 \mathrm{~mm}$ & \\
\hline $10-14$ & 1.00 & 1.00 & 1.00 & 1.00 & 0.97 & 1.00 & 0.99 \\
$15-19$ & 1.00 & 0.96 & 0.96 & 1.00 & 1.00 & 1.00 & 0.99 \\
$20-24$ & 1.00 & 0.92 & 0.93 & 1.00 & 1.00 & 0.97 & 0.97 \\
$25-29$ & 0.86 & 1.00 & 1.00 & 0.98 & 1.00 & 0.98 & 0.97 \\
$30-34$ & 1.00 & 0.93 & 1.00 & 0.92 & 0.97 & 0.97 & 0.96 \\
$35-39$ & 0.81 & 0.76 & 1.00 & 0.98 & 1.00 & 1.00 & 0.93 \\
$40-44$ & 0.48 & 0.79 & 0.98 & 0.94 & 1.00 & 1.00 & 0.86 \\
$45-51$ & 0.41 & 0.64 & 0.94 & 1.00 & 1.00 & 1.00 & 0.83 \\
Average & 0.77 & 0.86 & 0.97 & 0.98 & 0.99 & 0.99 & \\
\hline
\end{tabular}

The overall performance of the method is $91 \%$, as detailed in Table 4.5 , which shows the combined efficiency of CBD and SVM. 
Table 4.5. Overall accuracy.

\begin{tabular}{cccccccc}
\hline \multirow{2}{*}{ Vertical angle $\left(^{\circ}\right)$} & \multicolumn{7}{c}{ Loosened length } \\
& Tight & $5 \mathrm{~mm}$ & $10 \mathrm{~mm}$ & $15 \mathrm{~mm}$ & $20 \mathrm{~mm}$ & $25 \mathrm{~mm}$ & \\
\hline $10-14$ & 0.56 & 1.00 & 0.94 & 0.97 & 0.88 & 0.70 & 0.85 \\
$15-19$ & 0.93 & 0.86 & 0.85 & 0.96 & 0.92 & 0.74 & 0.89 \\
$20-24$ & 0.95 & 0.92 & 0.93 & 1.00 & 1.00 & 0.93 & 0.96 \\
$25-29$ & 0.83 & 1.00 & 1.00 & 0.98 & 1.00 & 0.98 & 0.97 \\
$30-34$ & 1.00 & 0.93 & 1.00 & 0.92 & 0.97 & 0.97 & 0.96 \\
$35-39$ & 0.81 & 0.76 & 1.00 & 0.98 & 1.00 & 1.00 & 0.93 \\
$40-44$ & 0.48 & 0.79 & 0.98 & 0.94 & 1.00 & 0.97 & 0.86 \\
$45-51$ & 0.35 & 0.64 & 0.94 & 1.00 & 1.00 & 1.00 & 0.82 \\
Average & 0.77 & 0.85 & 0.96 & 0.97 & 0.97 & 0.93 & \\
\hline
\end{tabular}

\subsection{Discussions}

The previous methods [51-53] are the only existing vision-based methods for loosened bolt detection. The former method [51-52] is suitable for angular bolts; it measures changes in angles due to the rotation of the bolt. The latter [53] is suitable for round bolts with wrench holes to measure the bolt features, using multilevel de-noising to crop localized bolts. Cha et. al. [53] used only one loosened length for the classification of bolts. However, the proposed method automatically localizes bolt images, crops the bolts using $\mathrm{CBD}$, and can be trained for any bolt shape. Therefore, it removes the bolt head shape limitation. Additionally, the current methodology removes the image multilevel de-noising and cropping processes by using contrast adjustment to 
improve the computational efficiency and loosened bolt detection rate. The proposed method was also tested on a broader range of loosened lengths (i.e. $5 \mathrm{~mm}$ to $25 \mathrm{~mm}$ ) to test the robustness of the method. The proposed method detected and classified the bolts from four horizontal angles (intervals of $30^{\circ}$ ). This demonstrated that varying the horizontal angle can accommodate different bolt layouts for detection and classification.

Although the method detects different shapes and orientations of bolts, there are some limitations. First, the computational time for training the cascade detector is slightly longer depending on the resolution and size of the training data and may go up to an hour or more. This is not a major drawback since the training has to be done only once for similar kinds of bolts, making it a onetime consumption in terms of time. Second, sharp camera angles still remain a limitation for SVM, whereas $\mathrm{CBD}$ is sensitive to low vertical angles. In Figure 4.7(a), a $5 \mathrm{~mm}$ loosened bolt is detected as tight because of the sharp angle; this is due to the small margin between the feature $h$ and $5 \mathrm{~mm}$ tight bolts, as mentioned previously. Also, in Figure 4.7(b), the bolt outlined in the highlighted image is not detected due to overlapping. Although vertical angles are improved when compared to the previous method, the horizontal camera angle is advantageous when countering the vertical angle issues in CBD detection. Third, as a vision-based approach, reflection and shadow play a major role both in detection and feature extraction. However, this can be avoided by using the camera at different angles and times of day. Finally, the training dataset plays an important role for $\mathrm{CBD}$ and SVM. For CBD, a negative dataset with a similar background of bolts improves the detection percentage and reduces the training size of training data. If similar background images are not available, this limitation can be countered by using a larger training dataset. For SVM training, bolt images with accurate extracted features should be used. The proposed method is 
effective for visual detection of the bolts (i.e. loosened or tightened), but measuring the mechanical properties of the bolted connections remains a limitation.

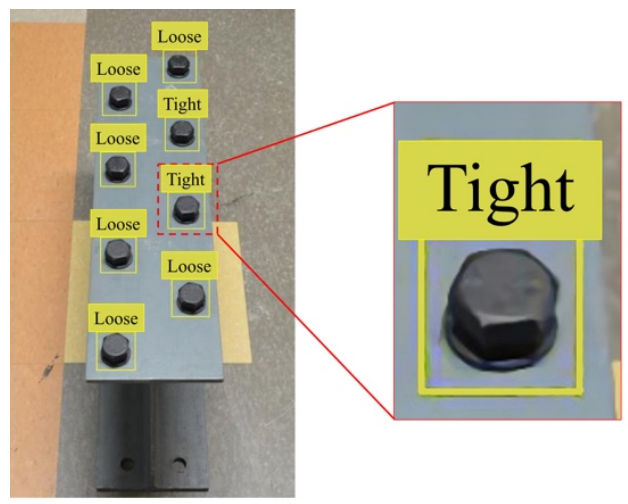

(a)

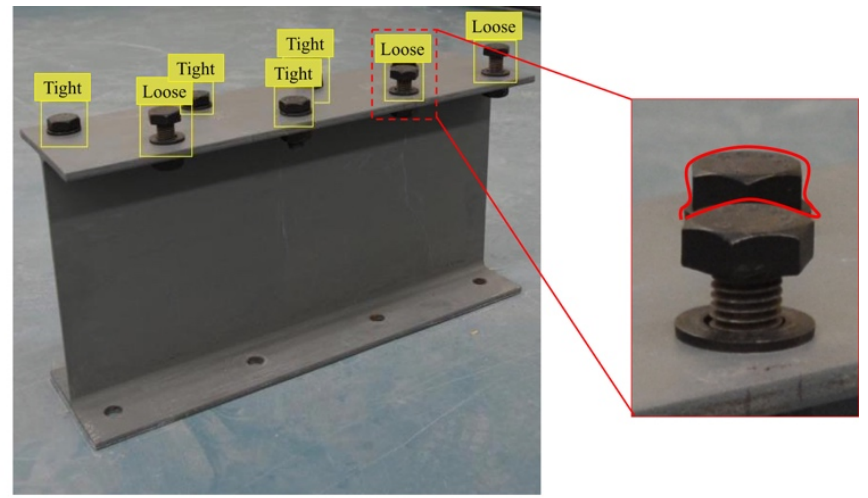

(b)

Figure 4.7. (a) False classification by SVM due to sharp vertical angle. (b) No bolt detection by CBD due to overlapping at low vertical angle. 


\section{Chapter 5 - Summary, Conclusions and Recommendations}

\subsection{Summary}

A damage detection method was developed based on a Viola-Jones algorithm and SVM. The study offers an automated vision-based method for bolt detection and evaluates the performance in terms of accuracy. First, the CBD was trained with a positive and negative image dataset to detect and crop the isolated bolt images using HOG features. The CBD was trained using the Adaboost algorithm. Second, the SVM was trained on the ratios of three features of loose and tight bolt image, using image processing to build a classification frontier for loosened and tight bolts. The training datasets for CBD and SVM were different from the test images and were not used for performance evaluation. The CBD detect bolts in the test images using a detection window at increasing scale and isolated bolt images are cropped. The cropped bolt images provided by the CBD were classified into loosened or tight based on bolt features extracted by the trained SVM. The images for the cascade detector and the SVM training were taken using smart phone cameras with an average resolution of $1024 \times 768$ and $90 \times 100$ pixels, respectively, whereas test images were taken using a Nikon D5200 DSLR with a resolution of $4000 \times 6000$ pixels.

\subsection{Conclusions}

The experiment was performed on 198 images and the vertical angle of every bolt calculated, as tabulated in Table 4.2. The accuracy of the cascade detector and SVM were measured individually along with the overall accuracy. The accuracy of the SVM was independent of errors in the cascade 
detector. The average detection accuracy of the cascade, SVM, and overall accuracy is $97 \%, 93 \%$, and $91 \%$, respectively.

During the experiment, the pros and cons of this method compared to existing vision-based loosened bolt detection were revealed as:

- Applicable to different shapes of bolt heads unlike the previous methods [51-53], which were only applicable to angular bolts and bolts with wrench holes in bolt head

- The horizontal and vertical angles can take care of a complex bolt layout geometry on structures. A bolted connection can occur in dense geometry, therefore varying angles can overcome the overlapping and isolated view of bolts

- Like previous vision-based methods, the method overcame environmental conditions when making damage detections since the data cannot be altered by environmental effects, which is also a motivation behind the vision-based methods

However, there are also limitations to the proposed method:

- The mechanical properties of connections cannot be measured. No vision-based method exists to the date that can measure torque of the bolted connection

- Reflections and shadows effect bolt detection and classification. Both CBD and SVM are sensitive to image noise. The CBD use HOG features and uses intensity values of pixels, hence, shadows can lead to the wrong detections; whereas, the SVM binarizes the image for feature extraction and is affected by shadows and reflections. The images must be taken carefully to avoid shadows, which is doable 
- Images with an exposed shank length that is hidden under the bolt head due to a sharp shooting angle is a limitation to this approach. Moreover, the small loosened lengths are more sensitive to sharp angles. The optimal range for best results is a $26^{\circ}-42^{\circ}$ vertical angle

\subsection{Future recommendations}

The limitations discussed in this study provide an opportunity for further exploration of this method and future research should:

- Explore detecting other type of damages in steel structures, since the cascade algorithm provides three type of feature descriptors

- Improve on the feature calculation and detection methods. The image noise effect on CBD and SVM can be further explored and improved for more robust performance

- Explore the prime area of this study - SVM sensitivity to the loosened length - as a progressive area of development 


\section{References}

[1] Balageas D, Fritzen CP, Güemes A, editors. Structural health monitoring. John Wiley \& Sons; 2010 Jan 5.

[2] 2016 Canadian Infrastructure report card. 2016. Retrieved June 17, 2017, from (http://canadianinfrastructure.ca/downloads/Canadian_Infrastructure Report 2016.pdf\#pag $\underline{\mathrm{e}=32)}$.

[3] 2013 Report Card for American Infrastructure. 2013. Retrieved February 19, 2017, from (http://www.infrastructurereportcard.org).

[4] Kiremidjian AS, Straser EG, Meng T, Law K, Soon H. Structural damage monitoring for civil structures. Proceedings of Structural Health Monitoring: Current Status and Perspectives. 1997:492-501.

[5] Rytter A. Vibrational based inspection of civil engineering structures. 1993

[6] Wang J, Nanukuttan SV, Basheer PA, Bai Y. Influence of micro and macro cracks due to sustained loading on chloride-induced corrosion of reinforced concrete beams. 2014

[7] How can one identify structural cracks in a concrete construction [Internet], from: https://www.quora.com/How-can-one-identify-structural-cracks-in-a-concrete-construction.

[8] Parking Facility - Underground Multi Family Residential [Internet]. http://grgas.ca., from: http://grgas.ca/portfolio/parking-facility-underground-multi-family-residential/.

[9] Travnicek RH. These photos speak volumes about bridge safety. 2013 Aug 22; 
from:http://www.revelstokecurrent.com/2013/08/22/these-photos-speak-volumes-aboutbridge-safety/.

[10] Structural - steel bolting [Internet]. 2014., from: http://www.epr-inc.com/blog/structuralsteel-bolting

[11] Phares BM, Rolander DD, Graybeal BA, Washer GA. Reliability of visual bridge inspection. Public Roads. 2001 Mar;64(5).

[12] Fan W, Qiao P. Vibration-based damage identification methods: a review and comparative study. Structural Health Monitoring. 2011 Jan 1;10(1):83-111.

[13] Salawu OS. Detection of structural damage through changes in frequency: a review. Engineering structures. 1997 Sep 1;19(9):718-23.

[14] Li J, Deng J, Xie W. Damage detection with streamlined structural health monitoring data. Sensors. 2015 Apr 15;15(4):8832-51.

[15] Wang T, Song G, Liu S, Li Y, Xiao H. Review of bolted connection monitoring. International Journal of Distributed Sensor Networks. 2013 Dec 1;9(12):871213.

[16] Epp T, Cha YJ. Air-coupled impact-echo damage detection in reinforced concrete using wavelet transforms. Smart Materials and Structures. 2016 Dec 30;26(2):025018.

[17] Cha YJ, Trocha P, Büyüköztürk O. Field Measurement-Based System Identification and Dynamic Response Prediction of a Unique MIT Building. Sensors. 2016 Jul 1;16(7):1016.

[18] Cha YJ, Wang Z. Unsupervised novelty detection-based structural damage localization using 
a density peaks-based fast clustering algorithm. Structural Health Monitoring. 2017 Feb 13:1475921717691260.

[19] Soh CK, Tseng KK, Bhalla S, Gupta A. Performance of smart piezoceramic patches in health monitoring of a RC bridge. Smart materials and Structures. 2000 Aug;9(4):533.

[20] Wang CS, Wu F, Chang FK. Structural health monitoring from fiber-reinforced composites to steel-reinforced concrete. Smart materials and Structures. 2001 Jun;10(3):548.

[21] Song G, Gu H, Mo YL, Hsu TT, Dhonde H. Concrete structural health monitoring using embedded piezoceramic transducers. Smart Materials and Structures. 2007 May 29;16(4):959.

[22] Mekjavić I. Identification of structural damage in bridges using high-frequency vibrational responses. Shock and Vibration. 2015 Sep 10;2015.

[23] Kim JT, Ryu YS, Cho HM, Stubbs N. Damage identification in beam-type structures: frequency-based method vs mode-shape-based method. Engineering structures. 2003 Jan $31 ; 25(1): 57-67$.

[24] Park S, Ahmad S, Yun CB, Roh Y. Multiple crack detection of concrete structures using impedance-based structural health monitoring techniques. Experimental Mechanics. 2006 Oct 1;46(5):609-18.

[25] Teughels A, De Roeck G. Structural damage identification of the highway bridge Z24 by FE model updating. Journal of Sound and Vibration. 2004 Dec 6;278(3):589-610.

[26] Weng JH, Loh CH, Yang JN. Experimental study of damage detection by data-driven 
subspace identification and finite-element model updating. Journal of structural engineering. 2009 Jun 10;135(12):1533-44.

[27] Park S, Yun CB, Roh Y, Lee JJ. Health monitoring of steel structures using impedance of thickness modes at PZT patches. Smart Structures and Systems. 2005;1:339-53.

[28] Doyle D, Zagrai A, Arritt B, Çakan H. Damage detection in bolted space structures. Journal of Intelligent Material Systems and Structures. 2010 Feb;21(3):251-64.

[29] Chen M, Xu B. Bolted joint looseness damage detection using electromechanical impedance measurements by PZT sensors. InThird International Conference on Smart Materials and Nanotechnology in Engineering 2012 Apr 2 (pp. 840925-840925). International Society for Optics and Photonics.

[30] Sevillano E, Sun R, Perera R. Damage Detection Based on Power Dissipation Measured with PZT Sensors through the Combination of Electro-Mechanical Impedances and Guided Waves. Sensors. 2016 May 5;16(5):639.

[31] Huda F, Kajiwara I, Hosoya N, Kawamura S. Bolt loosening analysis and diagnosis by noncontact laser excitation vibration tests. Mechanical systems and signal processing. 2013 Nov 30;40(2):589-604.

[32] Moaveni B, Conte JP, Hemez FM. Uncertainty and sensitivity analysis of damage identification results obtained using finite element model updating. Computer- Aided Civil and Infrastructure Engineering. 2009 Jul 1;24(5):320-34.

[33] Fritzen CP, Jennewein D, Kiefer T. Damage detection based on model updating methods. 
Mechanical systems and signal processing. 1998 Jan 1;12(1):163-86.

[34] Xia Y, Chen B, Weng S, Ni YQ, \& Xu YL. Temperature effect on vibration properties of civil structures: A literature review and case studies. J Civ Struct Healt Monit, 2012; 2(1), 29-46.

[35] Li J, Deng J, Xie W. Damage detection with streamlined structural health monitoring data. Sensors. 2015 Apr 15;15(4):8832-51.

[36] Uhl T, Kohut P, Holak K, Krupinski K. Vision based condition assessment of structures. In Journal of Physics: Conference Series 2011 (Vol. 305, No. 1, p. 012043). IOP Publishing.

[37] Torok MM, Golparvar-Fard M, Kochersberger KB. Image-based automated 3D crack detection for post-disaster building assessment. Journal of Computing in Civil Engineering. 2013 Jun 11;28(5):A4014004.

[38] Yu SN, Jang JH, Han CS. Auto inspection system using a mobile robot for detecting concrete cracks in a tunnel. Automation in Construction. 2007 May 31;16(3):255-61.

[39] Yamaguchi T, Hashimoto S. Fast crack detection method for large-size concrete surface images using percolation-based image processing. Machine Vision and Applications. 2010 Aug 1;21(5):797-809.

[40] Chen LC, Shao YC, Jan HH, Huang CW, Tien YM. Measuring system for cracks in concrete using multi-temporal images. Journal of surveying engineering. 2006 May;132(2):77-82.

[41] Huang Y, Xu B. Automatic inspection of pavement cracking distress. Journal of Electronic Imaging. 2006 Jan 1;15(1):013017. 
[42] Torok MM, Golparvar-Fard M, Kochersberger KB. Image-based automated 3D crack detection for post-disaster building assessment. Journal of Computing in Civil Engineering. 2013 Jun 11;28(5):A4014004.

[43] Cha YJ, Choi W, Buyukozturk O. Deep learning-based crack damage detection using convolutional neural network". Computer-Aided Civil and Infrastructure Engineering. 2017;32(3):2013-4.

[44] Chen PH, Shen HK, Lei CY, Chang LM. Support-vector-machine-based method for automated steel bridge rust assessment. Automation in Construction. 2012 May 31;23:9-19.

[45] Bonnin-Pascual F, Ortiz A. Corrosion detection for automated visual inspection. Developments in Corrosion Protection. 2014;25:619-32.

[46] Rafajłowicz E, Wietrzych J, Rafajłowicz W. A Computer Vision System for Evaluation of High Temperature Corrosion Damages in Steam Boilers. InIntelligent Systems in Technical and Medical Diagnostics 2014 (pp. 391-402). Springer Berlin Heidelberg.

[47] Uhl T, Kohut P, Holak K, Krupinski K. Vision based condition assessment of structures. InJournal of Physics: Conference Series 2011 (Vol. 305, No. 1, p. 012043). IOP Publishing.

[48] Ye XW, Ni YQ, Wai TT, Wong KY, Zhang XM, Xu F. A vision-based system for dynamic displacement measurement of long-span bridges: algorithm and verification. Smart Structures and Systems. 2013 Sep 1;12(3-4):363-79.

[49] Grano A, Zinno R. A new low-cost displacements monitoring system based on Kinect sensor. Journal of Civil Structural Health Monitoring. 2015 Nov 1;5(5):727-33. 
[50] Cha YJ, Chen JG, Büyüköztürk O. Output-only computer vision based damage detection using phase-based optical flow and unscented Kalman filters. Engineering Structures. 2017 Feb 1;132:300-13.

[51] Park JH, Kim TH, Kim JT. Image-based Bolt-loosening Detection Technique of Bolt Joint in Steel Bridges. In 6th International Conference on Advances in Experimental Structural Engineering 2015.

[52] Park JH, Huynh TC, Choi SH, Kim JT. Vision-based technique for bolt-loosening detection in wind turbine tower. Wind and Structures. 2015;21(6):709-26.

[53] Cha YJ, You K, Choi W. Vision-based detection of loosened bolts using the Hough transform and support vector machines. Automation in Construction. 2016 Nov 30;71:181-8.

[54] Viola P, Jones MJ. Robust real-time face detection. International journal of computer vision. 2004 May 1;57(2):137-54.

[55] Gunn SR. Support vector machines for classification and regression. ISIS technical report. 1998 May 10;14:85-6.

[56] Papageorgiou CP, Oren M, Poggio T. A general framework for object detection. InComputer vision, 1998. sixth international conference on 1998 Jan 4 (pp. 555-562). IEEE.

[57] Ojala T, Pietikainen M, Maenpaa T. Multiresolution gray-scale and rotation invariant texture classification with local binary patterns. IEEE Transactions on pattern analysis and machine intelligence. $2002 \mathrm{Jul} ; 24(7): 971-87$.

[58] Ojala T, Pietikainen M, Harwood D. Performance evaluation of texture measures with 
classification based on Kullback discrimination of distributions. InPattern Recognition, 1994. Vol. 1-Conference A: Computer Vision \& Image Processing., Proceedings of the 12th IAPR International Conference on 1994 Oct 9 (Vol. 1, pp. 582-585). IEEE.

[59] Dalal N, Triggs B. Histograms of oriented gradients for human detection. InComputer Vision and Pattern Recognition, 2005. CVPR 2005. IEEE Computer Society Conference on 2005 Jun 25 (Vol. 1, pp. 886-893). IEEE.

[60] Tomasi C. Histograms of Oriented Gradients.

[61] Viola P, Jones M. Rapid object detection using a boosted cascade of simple features. In Computer Vision and Pattern Recognition, 2001. CVPR 2001. Proceedings of the 2001 IEEE Computer Society Conference on 2001 (Vol. 1, pp. I-I). IEEE.

[62] Cui D, Gu D, Cai H, Sun J. License plate detection algorithm based on gentle AdaBoost algorithm with a cascade structure. InRobotics and Biomimetics (ROBIO), 2009 IEEE International Conference on 2009 Dec 19 (pp. 1962-1966). IEEE.

[63] Hamdi N, Auhmani K, Hassani MM, Elkharki O. AN EFFICIENT GENTLE ADABOOSTBASED APPROACH FOR MAMMOGRAMS CLASSIFICATION. Journal of Theoretical and Applied Information Technology. 2015 Nov 1;81(1):138.

[64] Mathworks (2017a), Computer Vision System Toolbox (2017a)

https://www.mathworks.com/help/vision/ug/train-a-cascade-object-detector.html

[65] Schapire RE. Explaining adaboost. InEmpirical inference 2013 (pp. 37-52). Springer Berlin Heidelberg. 
[66] Heydarzadeh Y, Haghighat AT. An efficient face detection method using adaboost and facial parts. IJSSST. 1997;12(4).

[67] Rogers S, Girolami M. A first course in machine learning. CRC Press; 2016 Oct 14.

[68] Xie Y, Ji Q. A new efficient ellipse detection method. InPattern Recognition, 2002. Proceedings. 16th International Conference on 2002 (Vol. 2, pp. 957-960). IEEE.

[69] Yuen, H. K., et al. "Comparative study of Hough transform methods for circle finding." Image and vision computing 8.1 (1990): 71-77.

[70] Al-Ameen Z, Sulong G, Johar MG. Employing a suitable contrast enhancement technique as a pre-restoration adjustment phase for computed tomography medical images. SERSC International Journal of Bio-Science and Bio-Technology. 2013 Feb;5(1):73-80.

[71] Mathworks (2017a), Computer Vision System Toolbox (2017a) https://www.mathworks.com/help/images/ref/activecontour.html 
Appendix A: Detailed information vertical angle of each bolt and corresponding CBD and SVM classifications 


\section{Vertical angle of all the bolts}

\begin{tabular}{|c|c|c|c|c|c|c|}
\hline \multirow{2}{*}{$\begin{array}{c}\text { Vertical } \\
\text { angle }\end{array}$} & \multicolumn{7}{|c|}{ Loosened Length } \\
\hline $10^{\mathrm{o}}$ & 0 & $5 \mathrm{~mm}$ & $10 \mathrm{~mm}$ & $15 \mathrm{~mm}$ & $20 \mathrm{~mm}$ & $25 \mathrm{~mm}$ \\
\hline $11^{\mathrm{o}}$ & 0 & 1 & 1 & 0 & 0 & 1 \\
\hline $12^{\mathrm{o}}$ & 3 & 3 & 4 & 6 & 6 & 3 \\
\hline $13^{\mathrm{o}}$ & 5 & 6 & 7 & 11 & 11 & 5 \\
\hline $14^{\mathrm{o}}$ & 8 & 8 & 5 & 8 & 11 & 8 \\
\hline $15^{\mathrm{o}}$ & 10 & 8 & 5 & 7 & 5 & 4 \\
\hline $16^{\mathrm{o}}$ & 5 & 4 & 4 & 4 & 8 & 6 \\
\hline $17^{\mathrm{o}}$ & 3 & 5 & 5 & 12 & 7 & 4 \\
\hline $18^{\mathrm{o}}$ & 5 & 5 & 6 & 16 & 10 & 6 \\
\hline $19^{\mathrm{o}}$ & 5 & 7 & 6 & 11 & 6 & 7 \\
\hline $20^{\mathrm{o}}$ & 2 & 7 & 7 & 15 & 7 & 5 \\
\hline $21^{\mathrm{o}}$ & 6 & 4 & 3 & 4 & 4 & 6 \\
\hline $22^{\mathrm{o}}$ & 3 & 6 & 6 & 10 & 7 & 4 \\
\hline $23^{\mathrm{o}}$ & 2 & 4 & 4 & 8 & 5 & 5 \\
\hline $24^{\mathrm{o}}$ & 6 & 5 & 9 & 10 & 10 & 10 \\
\hline $25^{\mathrm{o}}$ & 7 & 6 & 4 & 9 & 11 & 11 \\
\hline $26^{\mathrm{o}}$ & 4 & 8 & 10 & 13 & 14 & 10 \\
\hline $27^{\mathrm{o}}$ & 8 & 8 & 6 & 6 & 6 & 2 \\
\hline $28^{\mathrm{o}}$ & 2 & 5 & 4 & 12 & 12 & 12 \\
\hline $29^{\mathrm{o}}$ & 8 & 7 & 5 & 9 & 10 & 8 \\
\hline $30^{\mathrm{o}}$ & 3 & 6 & 10 & 8 & 10 & 12 \\
\hline $31^{\mathrm{o}}$ & 8 & 8 & 6 & 6 & 4 & 3 \\
\hline $32^{\mathrm{o}}$ & 5 & 2 & 1 & 6 & 8 & 11 \\
\hline $33^{\mathrm{o}}$ & 5 & 8 & 9 & 7 & 8 & 4 \\
\hline $34^{\mathrm{o}}$ & 2 & 5 & 7 & 10 & 6 & 9 \\
\hline $35^{\mathrm{o}}$ & 9 & 8 & 6 & 8 & 6 & 6 \\
\hline $36^{\mathrm{o}}$ & 5 & 4 & 5 & 7 & 9 & 9 \\
\hline $37^{\mathrm{o}}$ & 6 & 10 & 9 & 15 & 4 & 6 \\
\hline $38^{\mathrm{o}}$ & 7 & 4 & 6 & 7 & 4 & 6 \\
\hline $39^{\mathrm{o}}$ & 5 & 7 & 7 & 7 & 6 & 9 \\
\hline $40^{\mathrm{o}}$ & 10 & 16 & 13 & 8 & 9 & 10 \\
\hline $41^{\mathrm{o}}$ & 6 & 5 & 6 & 9 & 5 & 9 \\
\hline $42^{\mathrm{o}}$ & 7 & 8 & 8 & 8 & 11 & 9 \\
\hline $43^{\mathrm{o}}$ & 10 & 14 & 13 & 6 & 8 & 6 \\
\hline $44^{\mathrm{o}}$ & 7 & 4 & 4 & 5 & 4 & 4 \\
\hline $45^{\mathrm{o}}$ & 8 & 8 & 10 & 9 & 7 & 11 \\
\hline $46^{\mathrm{o}}$ & 8 & 9 & 6 & 3 & 3 & 3 \\
\hline $47^{\mathrm{o}}$ & 2 & 4 & 5 & 3 & 6 & 8 \\
\hline $48^{\mathrm{o}}$ & 8 & 6 & 5 & 8 & 6 & 4 \\
\hline $49^{\mathrm{o}}$ & 5 & 4 & 3 & 1 & 1 & 2 \\
\hline $50^{\mathrm{o}}$ & 0 & 2 & 3 & 4 & 4 & 4 \\
\hline & & & & & & \\
\hline
\end{tabular}




\section{Number of bolts detected by CBD}

\begin{tabular}{|c|c|c|c|c|c|c|}
\hline \multirow{2}{*}{$\begin{array}{c}\text { Vertical } \\
\text { angle }\end{array}$} & \multicolumn{7}{|c|}{ Loosened Length } \\
\hline $10^{\mathrm{o}}$ & 0 & $5 \mathrm{~mm}$ & $10 \mathrm{~mm}$ & $15 \mathrm{~mm}$ & $20 \mathrm{~mm}$ & $25 \mathrm{~mm}$ \\
\hline $11^{\mathrm{o}}$ & 0 & 1 & 1 & 0 & 0 & 1 \\
\hline $12^{\mathrm{o}}$ & 2 & 3 & 4 & 6 & 5 & 2 \\
\hline $13^{\mathrm{o}}$ & 3 & 6 & 7 & 10 & 10 & 5 \\
\hline $14^{\mathrm{o}}$ & 4 & 8 & 4 & 8 & 10 & 4 \\
\hline $15^{\mathrm{o}}$ & 9 & 8 & 5 & 6 & 5 & 2 \\
\hline $16^{\mathrm{o}}$ & 4 & 3 & 3 & 3 & 6 & 3 \\
\hline $17^{\mathrm{o}}$ & 3 & 4 & 5 & 12 & 7 & 3 \\
\hline $18^{\mathrm{o}}$ & 5 & 5 & 5 & 16 & 9 & 5 \\
\hline $19^{\mathrm{o}}$ & 5 & 6 & 5 & 11 & 6 & 7 \\
\hline $20^{\mathrm{o}}$ & 2 & 7 & 7 & 15 & 7 & 5 \\
\hline $21^{\mathrm{o}}$ & 6 & 4 & 3 & 4 & 4 & 5 \\
\hline $22^{\mathrm{o}}$ & 2 & 6 & 6 & 10 & 7 & 4 \\
\hline $23^{\mathrm{o}}$ & 2 & 4 & 4 & 8 & 5 & 5 \\
\hline $24^{\mathrm{o}}$ & 6 & 5 & 9 & 10 & 10 & 10 \\
\hline $25^{\mathrm{o}}$ & 6 & 6 & 4 & 9 & 11 & 11 \\
\hline $26^{\mathrm{o}}$ & 4 & 8 & 10 & 13 & 14 & 10 \\
\hline $27^{\mathrm{o}}$ & 8 & 8 & 6 & 6 & 6 & 2 \\
\hline $28^{\mathrm{o}}$ & 2 & 5 & 4 & 12 & 12 & 12 \\
\hline $29^{\mathrm{o}}$ & 8 & 7 & 5 & 9 & 10 & 8 \\
\hline $30^{\mathrm{o}}$ & 3 & 6 & 10 & 8 & 10 & 12 \\
\hline $31^{\mathrm{o}}$ & 8 & 8 & 6 & 6 & 4 & 3 \\
\hline $32^{\mathrm{o}}$ & 5 & 2 & 1 & 6 & 8 & 11 \\
\hline $33^{\mathrm{o}}$ & 5 & 8 & 9 & 7 & 8 & 4 \\
\hline $34^{\mathrm{o}}$ & 2 & 5 & 7 & 10 & 6 & 9 \\
\hline $35^{\mathrm{o}}$ & 9 & 8 & 6 & 8 & 6 & 6 \\
\hline $36^{\mathrm{o}}$ & 5 & 4 & 5 & 7 & 9 & 9 \\
\hline $37^{\mathrm{o}}$ & 6 & 10 & 9 & 15 & 4 & 6 \\
\hline $38^{\mathrm{o}}$ & 7 & 4 & 6 & 7 & 4 & 6 \\
\hline $39^{\mathrm{o}}$ & 5 & 7 & 7 & 7 & 6 & 9 \\
\hline $40^{\mathrm{o}}$ & 10 & 16 & 13 & 8 & 9 & 10 \\
\hline $41^{\mathrm{o}}$ & 6 & 5 & 6 & 9 & 5 & 9 \\
\hline $42^{\mathrm{o}}$ & 7 & 8 & 8 & 8 & 11 & 8 \\
\hline $43^{\mathrm{o}}$ & 10 & 14 & 13 & 6 & 8 & 6 \\
\hline $44^{\mathrm{o}}$ & 7 & 4 & 4 & 5 & 4 & 4 \\
\hline $45^{\mathrm{o}}$ & 8 & 8 & 10 & 9 & 7 & 11 \\
\hline $46^{\mathrm{o}}$ & 6 & 9 & 6 & 3 & 3 & 3 \\
\hline $47^{\mathrm{o}}$ & 1 & 4 & 5 & 3 & 6 & 8 \\
\hline $48^{\mathrm{o}}$ & 8 & 6 & 5 & 8 & 6 & 4 \\
\hline $49^{\mathrm{o}}$ & 4 & 4 & 3 & 1 & 1 & 2 \\
\hline $50^{\mathrm{o}}$ & 0 & 2 & 3 & 4 & 4 & 4 \\
\hline & & & & & & \\
\hline
\end{tabular}


True classification by SVM out of detected bolts by CBD

\begin{tabular}{|c|c|c|c|c|c|c|}
\hline \multirow{2}{*}{$\begin{array}{c}\text { Vertical } \\
\text { angle }\end{array}$} & \multicolumn{7}{|c|}{ Loosened Length } \\
\hline $10^{\mathrm{o}}$ & 0 & $5 \mathrm{~mm}$ & $10 \mathrm{~mm}$ & $15 \mathrm{~mm}$ & $20 \mathrm{~mm}$ & $25 \mathrm{~mm}$ \\
\hline $11^{\mathrm{o}}$ & 0 & 1 & 1 & 0 & 0 & 1 \\
\hline $12^{\mathrm{o}}$ & 2 & 3 & 4 & 6 & 5 & 2 \\
\hline $13^{\mathrm{o}}$ & 3 & 6 & 7 & 10 & 10 & 5 \\
\hline $14^{\mathrm{o}}$ & 4 & 8 & 4 & 8 & 9 & 4 \\
\hline $15^{\mathrm{o}}$ & 9 & 8 & 5 & 6 & 5 & 2 \\
\hline $16^{\mathrm{o}}$ & 4 & 3 & 3 & 3 & 6 & 3 \\
\hline $17^{\mathrm{o}}$ & 3 & 3 & 4 & 12 & 7 & 3 \\
\hline $18^{\mathrm{o}}$ & 5 & 5 & 5 & 16 & 9 & 5 \\
\hline $19^{\mathrm{o}}$ & 5 & 6 & 5 & 11 & 6 & 7 \\
\hline $20^{\mathrm{o}}$ & 2 & 7 & 7 & 15 & 7 & 4 \\
\hline $21^{\mathrm{o}}$ & 6 & 3 & 3 & 4 & 4 & 5 \\
\hline $22^{\mathrm{o}}$ & 2 & 5 & 5 & 10 & 7 & 4 \\
\hline $23^{\mathrm{o}}$ & 2 & 4 & 4 & 8 & 5 & 5 \\
\hline $24^{\mathrm{o}}$ & 6 & 5 & 8 & 10 & 10 & 10 \\
\hline $25^{\mathrm{o}}$ & 5 & 6 & 4 & 9 & 11 & 10 \\
\hline $26^{\mathrm{o}}$ & 4 & 8 & 10 & 13 & 14 & 10 \\
\hline $27^{\mathrm{o}}$ & 7 & 8 & 6 & 6 & 6 & 2 \\
\hline $28^{\mathrm{o}}$ & 2 & 5 & 4 & 11 & 12 & 12 \\
\hline $29^{\mathrm{o}}$ & 6 & 7 & 5 & 9 & 10 & 8 \\
\hline $30^{\mathrm{o}}$ & 3 & 6 & 10 & 8 & 9 & 12 \\
\hline $31^{\mathrm{o}}$ & 8 & 7 & 6 & 6 & 4 & 3 \\
\hline $32^{\mathrm{o}}$ & 5 & 2 & 1 & 6 & 8 & 11 \\
\hline $33^{\mathrm{o}}$ & 5 & 8 & 9 & 7 & 8 & 3 \\
\hline $34^{\mathrm{o}}$ & 2 & 4 & 7 & 7 & 6 & 9 \\
\hline $35^{\mathrm{o}}$ & 9 & 7 & 6 & 8 & 6 & 6 \\
\hline $36^{\mathrm{o}}$ & 4 & 3 & 5 & 7 & 9 & 9 \\
\hline $37^{\mathrm{o}}$ & 4 & 7 & 9 & 14 & 4 & 6 \\
\hline $38^{\mathrm{o}}$ & 4 & 4 & 6 & 7 & 4 & 6 \\
\hline $39^{\mathrm{o}}$ & 5 & 4 & 7 & 7 & 6 & 9 \\
\hline $40^{\mathrm{o}}$ & 4 & 12 & 13 & 7 & 9 & 10 \\
\hline $41^{\mathrm{o}}$ & 3 & 4 & 6 & 9 & 5 & 9 \\
\hline $42^{\mathrm{o}}$ & 4 & 5 & 7 & 7 & 11 & 8 \\
\hline $43^{\mathrm{o}}$ & 5 & 13 & 13 & 6 & 8 & 6 \\
\hline $44^{\mathrm{o}}$ & 3 & 3 & 4 & 5 & 4 & 4 \\
\hline $45^{\mathrm{o}}$ & 3 & 5 & 9 & 9 & 7 & 11 \\
\hline $46^{\mathrm{o}}$ & 4 & 6 & 6 & 3 & 3 & 3 \\
\hline $47^{\mathrm{o}}$ & 0 & 4 & 5 & 3 & 6 & 8 \\
\hline $48^{\mathrm{o}}$ & 2 & 2 & 5 & 8 & 6 & 4 \\
\hline $49^{\mathrm{o}}$ & 2 & 3 & 2 & 1 & 1 & 2 \\
\hline $50^{\mathrm{o}}$ & 0 & 1 & 3 & 4 & 4 & 4 \\
\hline & & & & & & \\
\hline
\end{tabular}


False classifications by SVM out of detected bolts by CBD

\begin{tabular}{|c|c|c|c|c|c|c|}
\hline \multirow{2}{*}{$\begin{array}{c}\text { Vertical } \\
\text { angle }\end{array}$} & \multicolumn{6}{|c|}{ Loosened Length } \\
\hline & $\mathrm{mm}$ & $5 \mathrm{~mm}$ & $10 \mathrm{~mm}$ & $15 \mathrm{~mm}$ & $20 \mathrm{~mm}$ & $25 \mathrm{~mm}$ \\
\hline $10^{\circ}$ & 0 & 0 & 0 & 0 & 0 & 0 \\
\hline $11^{\circ}$ & 0 & 0 & 0 & 0 & 0 & 0 \\
\hline $12^{\circ}$ & 0 & 0 & 0 & 0 & 0 & 0 \\
\hline $13^{\circ}$ & 0 & 0 & 0 & 0 & 0 & 0 \\
\hline $14^{\circ}$ & 0 & 0 & 0 & 0 & 1 & 0 \\
\hline $15^{\circ}$ & 0 & 0 & 0 & 0 & 0 & 0 \\
\hline $16^{\circ}$ & 0 & 0 & 0 & 0 & 0 & 0 \\
\hline $17^{\circ}$ & 0 & 1 & 1 & 0 & 0 & 0 \\
\hline $18^{\circ}$ & 0 & 0 & 0 & 0 & 0 & 0 \\
\hline $19^{\circ}$ & 0 & 0 & 0 & 0 & 0 & 0 \\
\hline $20^{\circ}$ & 0 & 0 & 0 & 0 & 0 & 1 \\
\hline $21^{\circ}$ & 0 & 1 & 0 & 0 & 0 & 0 \\
\hline $22^{\circ}$ & 0 & 1 & 1 & 0 & 0 & 0 \\
\hline $23^{\circ}$ & 0 & 0 & 0 & 0 & 0 & 0 \\
\hline $24^{\circ}$ & 0 & 0 & 1 & 0 & 0 & 0 \\
\hline $25^{\circ}$ & 1 & 0 & 0 & 0 & 0 & 1 \\
\hline $26^{\circ}$ & 0 & 0 & 0 & 0 & 0 & 0 \\
\hline $27^{\circ}$ & 1 & 0 & 0 & 0 & 0 & 0 \\
\hline $28^{\circ}$ & 0 & 0 & 0 & 1 & 0 & 0 \\
\hline $29^{\circ}$ & 2 & 0 & 0 & 0 & 0 & 0 \\
\hline $30^{\circ}$ & 0 & 0 & 0 & 0 & 1 & 0 \\
\hline $31^{\circ}$ & 0 & 1 & 0 & 0 & 0 & 0 \\
\hline $32^{\circ}$ & 0 & 0 & 0 & 0 & 0 & 0 \\
\hline $33^{\circ}$ & 0 & 0 & 0 & 0 & 0 & 1 \\
\hline $34^{\circ}$ & 0 & 1 & 0 & 3 & 0 & 0 \\
\hline $35^{\circ}$ & 0 & 1 & 0 & 0 & 0 & 0 \\
\hline $36^{\circ}$ & 1 & 1 & 0 & 0 & 0 & 0 \\
\hline $37^{\circ}$ & 2 & 3 & 0 & 1 & 0 & 0 \\
\hline $38^{\circ}$ & 3 & 0 & 0 & 0 & 0 & 0 \\
\hline $39^{\circ}$ & 0 & 3 & 0 & 0 & 0 & 0 \\
\hline $40^{\circ}$ & 6 & 4 & 0 & 1 & 0 & 0 \\
\hline $41^{\circ}$ & 3 & 1 & 0 & 0 & 0 & 0 \\
\hline $42^{\circ}$ & 3 & 3 & 1 & 1 & 0 & 0 \\
\hline $43^{\circ}$ & 5 & 1 & 0 & 0 & 0 & 0 \\
\hline $44^{\circ}$ & 4 & 1 & 0 & 0 & 0 & 0 \\
\hline $45^{\circ}$ & 5 & 3 & 1 & 0 & 0 & 0 \\
\hline $46^{\circ}$ & 2 & 3 & 0 & 0 & 0 & 0 \\
\hline $47^{\circ}$ & 1 & 0 & 0 & 0 & 0 & 0 \\
\hline $48^{\circ}$ & 6 & 4 & 0 & 0 & 0 & 0 \\
\hline $49^{\circ}$ & 2 & 1 & 1 & 0 & 0 & 0 \\
\hline $50^{\circ}$ & 0 & 1 & 0 & 0 & 0 & 0 \\
\hline
\end{tabular}

